\title{
Augmented Perpetual Manifolds and Perpetual Mechanical Systems-Part II: Theorem and a Corollary for Dissipative Mechanical Systems Behaving as Perpetual Machines
}

FOTIOS GEORGIADES ( $\nabla$ fotiosgeorgiades@citchennai.net)

Chennai Institute of Technology https://orcid.org/0000-0002-2784-6976

\section{Research Article}

Keywords: energy loss, mechanical engineering, internal damage, nonlinear dynamics

Posted Date: June 29th, 2021

DOI: https://doi.org/10.21203/rs.3.rs-647609/v1

License: (9) This work is licensed under a Creative Commons Attribution 4.0 International License. Read Full License 


\title{
Augmented perpetual manifolds and perpetual mechanical systems-Part II: Theorem and a corollary for dissipative mechanical systems behaving as perpetual machines
}

\author{
Fotios Georgiades \\ Centre for Nonlinear System, \\ Chennai Institute of Technology, Chennai, India \\ e-mail: fotiosgeorgiades@citchennai.net
}

ORCID ID: 0000-0002-2784-6976

\section{ABSTRACT}

Perpetual points in mathematics defined recently, and their significance in nonlinear dynamics and their application in mechanical systems is currently ongoing research. The perpetual points significance relevant to mechanics so far is that they form the perpetual manifolds of rigid body motions of mechanical systems. The concept of perpetual manifolds extended to the definition of augmented perpetual manifolds that an externally excited multi-degree of freedom mechanical system is moving as a rigid body. As a continuation of this work, herein the internal force's and their associated energies, for a motion of multi-degree of freedom dissipative flexible mechanical system with solutions in the exact augmented perpetual manifolds, leads to the proof of a theorem that based on a specific decomposition with respect to their state variables dependence, all the internal forcing vectors are equal to zero. Therefore there is no energy storage as potential energy, and the process is internally isentropic. This theorem provides the conditions that a mechanical system behaves as a perpetual machine of a $2^{\text {nd }}$ and a $3^{\text {rd }}$ kind. Then in a corollary, the behavior of a mechanical system as a perpetual machine of third kind further on is examined. The developed theory leads to a discussion for the conditions of the violation of the $2^{\text {nd }}$ law of thermodynamics for mechanical systems that their motion is described in the exact augmented perpetual manifolds. Moreover, the necessity of a reversible process to violate the 2 nd thermodynamics law, which is not valid, is shown. The findings of the theorem analytically and numerically are verified. The energies of a perpetual mechanical system in the exact augmented perpetual manifolds for two types of external forces have been determined. Then, in two examples of mechanical systems, all the analytical findings with numerical simulations, certified with excellent agreement. This work is essential in physics since the $2^{\text {nd }}$ law of thermodynamics is not admitting internally isentropic processes in the dynamics of dissipative mechanical systems. In mechanical engineering, the mechanical systems operating in exact augmented perpetual manifolds, with zero internal forces, there is no degradation of any internal part of the machine due to zero internal stresses. 
Also, the operation of a machine in the exact augmented perpetual manifolds is of extremely high significance to avoid internal damage, and there is no energy loss.

This article is dedicated to my educators, in all levels of my studies from school to post-doctoral researcher, including summer schools in Greece about Nonlinear dynamics and Chaos, naming few of them that cannot be found easily: Mr. Dimitris Aliapoulios, Prof. Michael Fridakis, Prof. Ajay Kapoor. (in chronological order)

Keywords: augmented perpetual manifolds; perpetual points; perpetual machines of second kind; perpetual machines of third kind;

\section{INTRODUCTION}

The perpetual points have been defined recently by Prasad in [1] as the sets of points that arise when the accelerations and jerks, describing the motion in a mechanical system, for non-zero velocity vector, are equal to zero. In [2], the experimental investigation of perpetual points in mechanical systems in a tilted pendulum is reported. So far, relevant to perpetual points, there are four main research directions. The first one is directly related to the perpetual points with the strict mathematical formulation of them [1,3-4], including the experimental research for identifying the perpetual points of mechanical systems [2]. In the second research direction, the perpetual points are used to advance nonlinear dynamics, such as locating hidden and chaotic attractors [5-13]. The third research direction is through perpetual points to identify dissipative systems [14-18] and the fourth one with their significance in mechanics [19-22].

This article is a continuation of the research relative to mechanics, that already, there are three theorems relevant to perpetual points, proved in [19-21]. The first two correlate the perpetual points of linear mechanical systems with rigid body motions, in [19] for conservative mechanical systems and in [20] for dissipative systems. Also, the perpetual points of mechanical systems that are forming perpetual manifolds in [20] is shown. In [21], based on the perpetual manifolds concept, the augmented perpetual manifolds are defined as those manifolds that arise in a multi-degrees of freedom flexible mechanical system that all the accelerations are equal but not necessarily zero. In the exact augmented perpetual manifolds, a multi-degree of freedom system is moving as a rigid body, since all 
inertia elements are having the same generalized coordinates. A theorem is proved by indicating the conditions that a solution of a nonlinear non-autonomous mechanical system can be in the exact augmented perpetual manifolds [21]. More precisely, the forces in the equations of motion are separated as internal forces and external forces. The internal forces correspond to a perpetual mechanical system by means this system admits exact rigid body motions as a solution. Then, the form of the external forces and initial conditions have been defined, such as the perpetual mechanical subsystem's motion is described by one ordinary differential equation with solutions in the exact augmented perpetual manifolds. The theorem's outcome leads to a corollary stating that the application of harmonic external forcing in a perpetual mechanical system leads to particle-wave motion. The standing and the longitudinal wave-particle motion of a multi-degrees of freedom mechanical system in [21] is shown. Another immediate outcome of the theorem in [21-22] is a corollary proved in [22] that in the exact augmented perpetual manifolds, the sum of the internal forces is zero.

This article is divided into two sections, the theoretical and the numerical section with the examples, and this work is a continuation of the work done in [21-22] by examining the internal forces and the energies at the exact augmented perpetual manifolds of natural mechanical systems incorporating the view of the 2nd law of Thermodynamics through the entropy definition given in [23].

In $\S 2.1$, the preliminary definitions, such as the relevant theory of the exact augmented perpetual manifolds developed in [21], separating the forces from internal and external and analysing them, lead to the definitions of the different types of energy of the natural mechanical system. Finally the entropies definitions for a closed system are shown.

In $\S 2.2$ a theorem is stated proved, by defining the conditions that all the individual internal forces, but not only as a sum that in [22] is shown, are zero when the exact augmented perpetual manifolds describe the dynamics of the perpetual mechanical system. Therefore dissipative forces too, which leads to noloss of internal energy as a heat to the environment which lead to a discussion about the existence of perpetual machines of $2^{\text {nd }}, 3^{\text {rd }}$ kind, and the validity of the 2nd law of thermodynamics, e.g., is it actual requirement the reversibility of the process, and the 'arrow of time' associated with the entropy, is discussed. 
As a note, for an out of the content, with the current article, science, the research of a pioneered team lead by Ilia Prigogine, examined the 2 nd law of thermodynamics for systems far away from thermodynamic equilibrium in chemistry [24].

In $\S 2.3$, a corollary is stated and proved, whether or not a mechanical system behaves as a perpetual machine of $3^{\text {rd }}$ kind in a reversible process.

In $\S 2.4$, the validity of the developed theory in sections 2.2 and 2.3 is examined analytically using two types of external forces. In section 2.4.1 the analytical determination of the different types of energies of a natural mechanical system in the exact augmented perpetual manifolds for the two types of external forcing is shown. In section 2.4.2, there is a preliminary analytical investigation, whether or not the two types of external forces lead to a perpetual machine of third kind behaviour.

The 2nd law of thermodynamics states that 'in a process, the entropy of a system is increasing' [23, 25-26].

In section 3, the analytical findings with two examples are numerically. The first example is a perpetual mechanical system, and the $2^{\text {nd }}$ example is a non-perpetual mechanical system, but within some boundaries, there is a perpetual mechanical subsystem, under conditions.

\section{THEORY}

\subsection{Preliminaries}

In some mechanical systems, the perpetual points are not just a few points, but they form the perpetual manifolds [19-20]. In [21], the exact perpetual manifolds of rigid body motions were defined as the sets of perpetual points that correspond to exact rigid body motions, whereas all masses have the same displacement. Moreover, in [21], the perpetual mechanical systems were defined as the unforced systems that admit exact perpetual manifolds solutions. Also, in [21], the concept of augmented perpetual manifolds is introduced, and these are manifolds defined by the solutions of the equations of the forced perpetual mechanical systems in the state-space when all the time depended accelerations have the same but not necessarily zero values. In forced systems, the solutions, that all the generalized velocities are the same and all the generalized coordinates are the same, form the 
exact augmented perpetual manifolds of rigid body motions. In mathematical form are defined as follows [21],

The $2 N+1$ dimensional Exact Augmented Perpetual Manifolds e.g.X of a $N$-dof mechanical discrete system, with generalized coordinates $q_{i}$, that admits solutions of perpetual manifolds, arise when,

$$
\ddot{q}_{i}(t)=\ddot{q}_{a}(t), \quad \text { for }=1, \ldots, N \text {, and } \ddot{q}_{a}(t) \in \mathbb{R},
$$

and the solutions of the system in state space, define them, as,

$$
X=\left\{\left(t, q_{a}(t), \ldots, q_{a}(t), \dot{q}_{a}(t), \ldots, \dot{q}_{a}(t)\right):\left(t, q_{a}(t), \dot{q}_{a}(t)\right) \in \mathbb{R}^{3}\right\} .
$$

whereas the overdot means the derivatives in time, and based on the above definitions, a theorem in [22] is written and in [21] is proven, and it is about the conditions that a mechanical system has a solution in an exact augmented perpetual manifold.

In this article, the developed theory is restricted to natural mechanical systems, and the theorem of [21-22] can be written as follows:

Any $N(\geq 2)$-degrees of freedom discrete natural perpetual mechanical systems with constant inertia matrix $-M_{i j}$, described by the following equations of motion,

$$
\begin{gathered}
{\left[M_{i, j}\right] \times\left\{\ddot{q}_{i}(t)\right\}+\left[C_{i, j}\right] \times\left\{\dot{q}_{i}(t)\right\}+\left[K_{i, j}\right] \times\left\{q_{i}(t)\right\}+} \\
+\left\{F_{i}^{N L}\left(q_{n}(t), \dot{q}_{o}(t)\right)\right\}=\left\{F_{i}\left(q_{s}(t), \dot{q}_{z}(t), t\right)\right\}, \text { for } \\
i=1, \ldots, N, j=1, \ldots, N, n, o, s, z \in\{1,2, \ldots, N\} \text { and } \\
\left(q_{i}(t), \dot{q}_{i}(t), \ddot{q}_{i}(t)\right) \in \mathbb{R}^{3},
\end{gathered}
$$

with the following property,

$$
\left[C_{i j}\right] \times\{1\} \cdot \dot{q}_{a}+\left[K_{i j}\right] \times\{1\} \cdot q_{a}+\left\{F_{N L, i}\left(q_{a}, \dot{q}_{a}\right)\right\}=\{0\}, \text { for }\left(q_{a}, \dot{q}_{a}\right) \in \mathbb{R}^{2},
$$

whereas, $K_{i j}$ and $C_{i j}$ are the linear stiffness and the proportional to the velocity vector matrices, respectively. The nonlinear forces vector $\left(F_{N L, i}\right)$ can have as elements nonsmooth functions but single-valued in the exact augmented perpetual manifolds, the external forces $\left(F_{i}\right)$ might be nonsmooth functions too.

The system's solution in the exact augmented perpetual manifolds of rigid body motion requires the following correlation of the external forces,

$$
\begin{aligned}
& F_{i}\left(t, q_{a}(t), \dot{q}_{a}(t)\right)=\frac{\sum_{j=1}^{N} M_{i, j} F_{k}\left(t, q_{a}(t), \dot{q}_{a}(t)\right)}{\sum_{j=1}^{N} M_{k, j}}, \quad \text { for } \\
& i, k \in\{1,2, \ldots, N\}, \text { and } q_{a}(t)=q_{i}(t), \dot{q}_{a}(t)=\dot{q}_{i}(t),
\end{aligned}
$$

that lead to the following characteristic differential equation, describing the motion, 


$$
\ddot{q}_{a}(t)=\frac{F_{k}\left(t, q_{a}(t), \dot{q}_{a}(t)\right)}{\sum_{j=1}^{N} M_{k, j}}=G\left(t, q_{a}(t), \dot{q}_{a}(t)\right),
$$

with vector field $G$, for a set of initial conditions, at the time instant $t_{0}$, given by equations,

$$
\begin{aligned}
& q_{i}\left(t_{0}\right)=q_{a}\left(t_{0}\right), \quad \text { for } \quad i=1, \ldots, N, \quad \text { and }, \quad q_{a}\left(t_{0}\right) \in \mathbb{R}, \\
& \dot{q}_{i}\left(t_{0}\right)=\dot{q}_{a}\left(t_{0}\right), \quad \text { for } \quad i=1, \ldots, N, \quad \text { and, } \quad \dot{q}_{a}\left(t_{0}\right) \in \mathbb{R},
\end{aligned}
$$

defines the generalized coordinates $-q_{i}$ and their velocities $-\dot{q}_{i}$ in the exact augmented perpetual manifold as described by equation (2).

The sign ' $x$ ' for multiplication of matrices is used, and the sign ${ }^{\prime} \cdot$ for the scalar product is used.

Forces analysis for the development of the theory

-The generalized coordinate's only dependent forces (linear and nonlinear), at least in the exact augmented perpetual manifolds, they must arise by an elastic potential. The nonlinear generalized coordinate's dependent forces, in their general form, since they can be nonsmooth functions, it might be impossible to be defined, even by using sub-differentials of an elastic potential function. In the exact augmented perpetual manifolds, they must be single-valued forces, and their elastic potential function becomes mathematically possible to be defined. -The existence of 'external' forces $-F_{i}$ that are on the right-hand side of equations (3) means that the perpetual natural mechanical system is not isolated, but it is a subsystem. These forces in the exact augmented perpetual manifolds either correspond to more 'grounded' (connected with rigid places) elements, e.g. springs, dashpots, etc., that build up the overall system or external forces of a field or any other source by the environment. Therefore the 'external' forces $-F_{i}$ are the forces that connect the perpetual natural mechanical subsystem with the 'environment'. The boundaries for perpetual mechanical system internal energy analysis are defined by its limits and are associated with the forces on the lefthand side of equations (3). These elements are not considered part of the perpetual natural mechanical subsystem because their consideration does not lead to a perpetual natural mechanical subsystem. Therefore these elements are treated as external forces, and they are separated on the right-hand side of equations (3). The sum of energies associated with the kinetic energies of the inertia elements and the energies obtained by integrating the power of the internal forces (the left side of equations of motion) comprises the internal energy of the perpetual 
mechanical subsystem. The rest are considered as energies associated with the external forces to this subsystem.

The energy of all the internal forces included in equation (4) in the exact augmented perpetual manifolds is given by integrating the internal forces power of the system, as follows:

$$
\begin{aligned}
& E_{\text {int }}=\int_{t_{0}}^{t}\left\{\left[C_{i j}\right] \times\{1\} \cdot \dot{q}_{a}(\tau)+\left[K_{i j}\right] \times\{1\} \cdot q_{a}(\tau)+\left\{F_{N L, i}\left(q_{a}(\tau), \dot{q}_{a}\right)\right\}\right\}^{T} \times\{1\} \\
& \cdot \dot{q}_{a}(\tau) d \tau=0=E_{L, \dot{q}}(t)+U_{L}\left(q_{i}(t)\right)+U_{N L}\left(q_{i}(t)\right)+E_{N L, \dot{q}}(t)+E_{N L, g}(t),
\end{aligned}
$$

which means that the sum of the energies of the different types of internal forces is equal to zero. Although the energies associated with the two types of linear internal forces in linear perpetual mechanical systems is shown in [20] that are zero, but this is not the case for the arbitrarily defined nonlinear forces since the energy associated with each one of them might change form between them with a sum equal to zero.

In order to understand clearly the energies associated with each type of nonlinear internal forces (might be nonsmooth too) of equation (4), the nonlinear internal forces are separated as follows,

$$
\begin{gathered}
\left\{F_{N L, i}\left(q_{n}(t), \dot{q}_{o}(t)\right)\right\}= \\
=\left\{F_{N L, q, i}\left(q_{l}(t)\right)\right\}+\left\{F_{N L, \dot{q}, i}\left(\dot{q}_{r}(t)\right)\right\}+\left\{F_{N L, g, i}\left(q_{k}(t), \dot{q}_{m}(t)\right)\right\}, \\
\text { with } k, l, m, n, o, r \in\{1,2, \ldots, N\},
\end{gathered}
$$

whereas, the first term is nonlinear generalized coordinates dependent forces and must be associated with potential energy, the second term are nonlinear generalized velocities depended forces and the third term being the generalized nonlinear forces with the condition that none part of the third term can be decomposed as a linear combination of any of the other two types of nonlinear forces.

In the following part of this section, the energies of natural mechanical systems with a motion described by equations (3) are defined as follows [27-29]:

-Kinetic energy, for smooth functions of velocities, is given by $\left(E_{T}\right)$,

$$
E_{T}\left(q_{i}(t), \dot{q}_{i}(t)\right)=E_{T_{2}}\left(\dot{q}_{i}(t)\right)=\frac{1}{2} \cdot\left\{\dot{q}_{i}(t)\right\}^{T} \times\left[M_{i j}\right] \times\left\{\dot{q}_{i}(t)\right\},
$$

whereas for natural mechanical systems is limited to the so-called " $T_{2}$ " kinetic energy [29].

-Potential energy $\left(U_{t o t}\right)$ of the perpetual mechanical system, 


$$
\begin{gathered}
U_{t o t}\left(q_{i}(t)\right)=U_{L}\left(q_{i}(t)\right)+U_{N L}\left(q_{i}(t)\right)= \\
=\frac{1}{2} \cdot\left\{q_{i}(t)\right\}^{T} \times\left[K_{i j}\right] \times\left\{q_{i}(t)\right\}+U_{N L}\left(q_{i}(t)\right),
\end{gathered}
$$

whereas, the first term is the potential energy associated with the linear forces. Since the nonlinear forces are not necessarily smooth functions, therefore the second term of the potential of the nonlinear forces in this general form might be impossible to be defined even through sub-differentials of forces Nevertheless, the restrictions of these types of forces, for a solution in the exact augmented perpetual manifolds, lead that these potentials can be defined.

-Energy $\left(E_{\dot{q}}\right)$ associated with the velocity dependent forces is given by [28],

$$
\begin{gathered}
E_{\dot{q}}(t)=E_{L, \dot{q}}(t)+E_{N L, \dot{q}}(t)= \\
=\int_{t_{0}}^{t}\left\{\left[C_{i j}\right] \times\left\{\dot{q}_{i}(\tau)\right\}\right\}^{T} \times\left\{\dot{q}_{i}(\tau)\right\} d \tau+\int_{t_{0}}^{t}\left\{F_{N L, \dot{q}, i}\left(\dot{q}_{r}(t)\right)\right\}^{T} \times\left\{\dot{q}_{i}(\tau)\right\} d \tau,(10 \mathrm{c})
\end{gathered}
$$

whereas, the first term corresponds to linear forces. This integral is not always defined in the general nonlinear and nonsmooth form of the velocities dependent nonlinear forces. This general case that the integral might not be defined is not a problem for developing this theory. The reason is that the examination of the dynamics is restricted in the exact augmented perpetual manifolds that certain restrictions of the functional form of these forces are given, and these restrictions lead to the existence of the integral of equation (10c). Upon each energy explicit functional form term, these forces, linear and nonlinear, might be dissipative or not (flutter) [28]. If any term of the energy of the generalized velocities dependent forces in equation (10c) is negative, then the system is gaining mechanical energy associated with this force (linear or nonlinear). If it is positive, the system is losing mechanical energy, and the associated forces are dissipative. The initial energy associated with this type of forces is related to the system's dynamics from previous time instants and represents the energy lost or gained in the system's mechanical energy, which is the reason that is disregarded.

- Energy of the generalized forces $\left(E_{g}\right)[28]$,

$$
\begin{aligned}
& E_{N L, g}(t)=\int_{t_{0}}^{t}\left\{F_{N L, g, i}\left(q_{k}(\tau), \dot{q}_{m}(\tau)\right)\right\}^{T} \times\left\{\dot{q}_{i}(\tau)\right\} d \tau+E_{g}\left(t_{0}\right)= \\
& =\int_{t_{0}}^{t}\left\{F_{N L, g, i}\left(q_{k}(\tau), \dot{q}_{m}(\tau)\right)\right\}^{T} \times\left\{\dot{q}_{i}(\tau)\right\} d \tau .
\end{aligned}
$$

Similarly, with the nonlinear generalized velocity-dependent forces, due to the force's general type, which might be nonsmooth, this integral is not always defined, e.g., at the force's discontinuities. Herein, the theory is developed around 
the augmented perpetual manifolds and lead to integrable functions in equation (10d). As mentioned previously, if the energy of generalized forces in equation (10d) is negative, then the system is gaining mechanical energy. In the case of being positive, the system loses mechanical energy, and they are dissipative forces. The generalized force's initial energy represents the mechanical energy gained or lost from the system's previous dynamic evolution and is disregarded. -Power $\left(P_{e x}\right)$, and the work done $\left(W_{e x}\right)$ by the external forces is given by [28],

$$
P_{e x}\left(q_{p}(t), \dot{q}_{q}(t), t\right)=\frac{d E_{e x}(t)}{d t}=\left\{F_{i}\left(q_{p}(t), \dot{q}_{q}(t), t\right)\right\}^{T} \times\left\{\dot{q}_{i}(t)\right\},
$$

and,

$$
\begin{gathered}
W_{e x}(t)=\int_{t_{0}}^{t} P_{e x}\left(q_{p}(\tau), \dot{q}_{q}(\tau), \tau\right) d \tau+W_{e x}\left(t_{0}\right)= \\
=\int_{t_{0}}^{t}\left\{F_{i}\left(q_{p}(\tau), \dot{q}_{q}(\tau), \tau\right)\right\}^{T} \times\left\{\dot{q}_{i}(\tau)\right\} d \tau+W_{e x}\left(t_{0}\right)= \\
=\int_{t_{0}}^{t}\left\{F_{i}\left(q_{p}(\tau), \dot{q}_{q}(\tau), \tau\right)\right\}^{T} \times\left\{\dot{q}_{i}(\tau)\right\} d \tau,
\end{gathered}
$$

whereas, for any functional form of the external forces, which might be nonsmooth functions, the integral of equation (10f) cannot always be defined, e.g. in time instants e.g. with such discontinuities that the integral given by the equation (10f) is not defined. The external force's initial energy is the energy gained or lost of the mechanical energy throughout previous time instances. That is the reason that is disregarded.

In the exact augmented perpetual manifolds, considering a solution in the form of equation (2), the kinetic energy is taking the form,

$$
\begin{gathered}
E_{T, a}\left(\dot{q}_{a}(t)\right)=\frac{1}{2} \cdot\left\{\dot{q}_{a}(t)\right\}^{T} \times\left[M_{i j}\right] \times\left\{\dot{q}_{a}(t)\right\}=\frac{1}{2} \cdot\{1\}^{T} \times\left[M_{i j}\right] \times\{1\} \cdot \dot{q}_{a}^{2}(t)= \\
=\frac{1}{2} \cdot \sum_{i=1}^{N} \sum_{j=1}^{N} M_{i, j} \cdot \dot{q}_{a}^{2}(t),
\end{gathered}
$$

The power of the external forces is defined by equation (10e), and considering a correlation of external forces given by equation (5), then is taking the form,

$$
\begin{gathered}
P_{e x, a}\left(q_{a}(t), \dot{q}_{a}(t), t\right)=\frac{d E_{e x, a}(t)}{d t}= \\
=\frac{F_{k}\left(t, q_{a}(t), \dot{q}_{a}(t)\right)}{\sum_{j=1}^{N} M_{k, j}} \cdot\left\{\sum_{j=1}^{N} M_{i, j}\right\}^{T} \times\{1\} \cdot \dot{q}_{a}(t)= \\
=\frac{\sum_{i=1}^{N} \sum_{j=1}^{N} M_{i, j}}{\sum_{j=1}^{N} M_{k, j}} \cdot F_{k}\left(q_{a}(t), \dot{q}_{a}(t), t\right) \cdot \dot{q}_{a}(t)=\frac{\sum_{i=1}^{N} \sum_{j=1}^{N} M_{i, j}}{\sum_{j=1}^{N} M_{k, j}} \cdot P_{e x, a, k}\left(q_{a}(t), \dot{q}_{a}(t), t\right),
\end{gathered}
$$


whereas the last term $\left(P_{e x, a, k}\right)$ is the power of the $k$-external force of the $k$-reference mass used in the derivation of the characteristic differential equation.

Considering equation (11b) in equation (10f) lead that the total work done by the external forces $\left(W_{e x, a}\right)$ is given by,

$$
W_{e x, a}(t)=\frac{\sum_{i=1}^{N} \sum_{j=1}^{N} M_{i, j}}{\sum_{j=1}^{N} M_{k, j}} \cdot W_{e x, a, k}(t),
$$

whereas the last term $\left(W_{e x, a, k}\right)$ is the work done by the $k$-external force of the $k$-reference mass.

The work done by the external forces may cause either decrease or increase of the system's energy, and at each time instant, the positive (negative) sign of the power associated with the external forces indicates if it is actually input (output) energy. The equations (3) for smooth forces can be easily derived using the least action principle, Newton's law or Lagrange equation. In a system with nonsmooth nonlinear forces (internal and external), the derivation of equations of motion through the least action principle has some limitations, and as representative constraint, the integrands must be nonsmooth but convex functions [30-32]. The mechanical system described by equations (3) is not necessarily a representation of a specific configuration of a mechanical structure but generally a system that the perspective of the theory of mechanics is applied, e.g. a solid-state lattice [33]. In that respect, the mechanical systems' dynamics in the exact augmented perpetual manifolds through thermodynamics is examined in the following section.

More precisely, the perpetual natural (without any gyroscopic effects) mechanical systems in the exact augmented perpetual manifolds, through the individual energies of the system, are examined by considering the 2nd law using the entropy definition [23].

In the following part of this section, some preliminary definitions from thermodynamics are introduced.

The perpetual mechanical systems are closed systems since the number of degrees of freedom remains constant [23,25-26].

In a process the total change of entropy $(\Delta S)$ of a system is comprised by two terms [23],

$$
\Delta S=\Delta S_{\text {ext }}+\Delta S_{\text {int }}
$$


whereas, the first term is associated with the entropy of the surroundings of the system, and for closed systems (perpetual mechanical subsystem adding the source of the external forces) is given by,

$$
\Delta S_{\text {ext }}=\frac{\Delta Q}{T},
$$

with $\Delta Q$ being the heat difference in an absolute temperature $(T)$. The difference of the surroundings entropy is zero for an adiabatic process.

The second term in equation (12a) is associated with the internal change of entropy,

$$
\Delta S_{\text {int }}=\frac{\Delta Q}{T} .
$$

The $2^{\text {nd }}$ law of thermodynamics through the entropy definition states that,

$$
\Delta S_{\text {int }} \geq 0
$$

and,

$$
\Delta S=\frac{\Delta Q}{T} \geq 0,
$$

with the equality to zero in case of reversible process.

In the considered mechanical configuration, the thermal environmental source of energy is not examined. Only the loss of mechanical energy in the form of heat due to dissipation within the perpetual mechanical system is examined, which leads to the examination of the internal entropy difference $\left(\Delta S_{\text {int }}\right)$ in a process described by the dynamics of a perpetual mechanical subsystem in the exact augmented perpetual manifolds.

In an environment of a perpetual mechanical system that the source of energy provides the external forces to the perpetual mechanical system through an adiabatic process which means that the entropy in the environment is constant, and in case that the internal entropy is constant, then the system behaves as a perpetual machine of 2 nd kind [23].

The perpetual machine of $3^{\text {rd }}$ kind is not related to the $2^{\text {nd }}$ law of thermodynamics [26]. The entropy of the surrounding environment is not considered but only the internal entropy of the system, and the perpetual machine of $3^{\text {rd }}$ kind is defined when there is no energy loss, e.g. due to friction in the mechanical system [26]. In the next section, a theorem and a corollary are proved for perpetual mechanical subsystems when the exact augmented perpetual manifolds define their motion. 


\subsection{Theorem}

In this section, the theoretical developments in [21-22] are extended by examining energies in the exact augmented perpetual manifolds of the perpetual natural mechanical systems. The examination of the individual energies, associated with each type of internal forces of a mechanical system, when its motion is described in the exact augmented perpetual manifolds, leads to the proof of a theorem.

Theorem

A N( $\geq 2)$-degrees of freedom perpetual natural mechanical discrete subsystem, with underlying perpetual linear mechanical system, described by the following equations of motion,

$$
\begin{gathered}
{\left[M_{i j}\right] \times\left\{\ddot{q}_{i}(t)\right\}+\left[C_{i j}\right] \times\left\{\dot{q}_{i}(t)\right\}+\left[K_{i j}\right] \times\left\{q_{i}(t)\right\}+\left\{F_{N L, q, i}\left(q_{l}(t)\right)\right\}+} \\
+\left\{F_{N L, \dot{q}, i}\left(\dot{q}_{r}(t)\right)\right\}+\left\{F_{N L, g, i}\left(q_{k}(t), \dot{q}_{m}(t)\right)\right\}=\left\{F_{i}\left(q_{s}(t), \dot{q}_{z}(t), t\right)\right\}, \quad \text { for } \\
i=1, \ldots, N, j=1, \ldots, N, k, l, m, r, s, z \in\{1,2, \ldots, N\}, \text { and } \\
\left(q_{i}(t), \dot{q}_{i}(t), \ddot{q}_{i}(t), t\right) \in \mathbb{R}^{4}
\end{gathered}
$$

whereas, the external forces are on the right-hand side of equation (13), the generalized coordinates dependent internal forces in the exact augmented perpetual manifolds are associated with an elastic potential functional; in the exact augmented perpetual manifolds the nonlinear internal force's vectors that can be decomposed as a sum of vectors, for any decomposition each vector is orthogonal to each other, any part of each element of $F_{N L, g, i}$ cannot be decomposed as a linear combination of the other two types of nonlinear forces, the dynamical system is solvable, for zero velocities the velocity depended nonlinear forces are vanishing, and the velocities are finite, then in the exact augmented perpetual manifolds:

a) Each individual internal forcing vector is equal zero.

b) This perpetual mechanical subsystem dynamic's evolution occurs with constant potential energy.

c) The process is internally isentropic.

d) In the appropriate environment that the external forces obtained through an adiabatic process, the mechanical configuration of the overall system is behaving as a perpetual machine of $2^{\text {nd }}$ kind.

e) In case that the characteristic differential equation is smooth or nonsmooth for the time interval that forms a Filippov's system, the work 
done by the external forces adding the initial kinetic energy, is equal to the kinetic energy of the system.

f) For the time interval that the external force is co-directed with the velocity of the generalized coordinate of the system then this subsystem behaves as a perpetual machine of $3^{\text {rd }}$ kind.

Proof:

In the exact augmented perpetual manifolds with a solution defined by the differential equation (6), the system of equations (13) is taking the form,

$$
\begin{aligned}
& {\left[M_{i, j}\right] \times\left\{\ddot{q}_{a}(t)\right\}+\left[C_{i, j}\right] \times\left\{\dot{q}_{a}(t)\right\}+\left[K_{i, j}\right] \times\left\{q_{a}(t)\right\}+\left\{F_{N L, q, i}\left(q_{a}(t)\right)\right\}+} \\
& \quad+\left\{F_{N L, \dot{q}, i}\left(\dot{q}_{a}(t)\right)\right\}+\left\{F_{N L, g, i}\left(q_{a}(t), \dot{q}_{a}(t)\right)\right\}=\left\{F_{i}\left(q_{a}(t), \dot{q}_{a}(t), t\right)\right\},(14 \mathrm{a})
\end{aligned}
$$

taking common factor the acceleration, and considering equations (5) lead to,

$$
\begin{aligned}
& {\left[M_{i, j}\right] \times\{1\} \cdot \ddot{q}_{a}(t)+\left[C_{i, j}\right] \times\left\{\dot{q}_{a}(t)\right\}+\left[K_{i, j}\right] \times\left\{q_{a}(t)\right\}+} \\
& +\left\{F_{i}^{N L}\left(q_{a}(t), \dot{q}_{a}(t)\right)\right\}=\frac{F_{k}\left(q_{a}(t), \dot{q}_{a}(t), t\right)}{\sum_{j=1}^{N} M_{k, j}} \cdot\left\{\sum_{j=1}^{N} M_{i, j}\right\} .
\end{aligned}
$$

Since the motion in the exact augmented perpetual manifolds is defined by equation (6) then equation (14b) is taking the final form,

$$
\begin{gathered}
\left\{\sum_{j=1}^{N} C_{i, j}\right\} \cdot \dot{q}_{a}(t)+\left\{\sum_{j=1}^{N} K_{i, j}\right\} \cdot q_{a}(t)+\left\{F_{N L, q, i}\left(q_{a}(t)\right)\right\}+\left\{F_{N L, \dot{q}, i}\left(\dot{q}_{a}(t)\right)\right\}+ \\
+\left\{F_{N L, g, i}\left(q_{a}(t), \dot{q}_{a}(t)\right)\right\}=\{0\}=\left\{\sum_{j=1}^{N} C_{i, j}\right\} \cdot \dot{q}_{a}(t)+\left\{\sum_{j=1}^{N} K_{i, j}\right\} \cdot q_{a}(t)+ \\
+\sum_{j=1}^{k}\left\{F_{N L, q, i, j}\left(q_{a}(t)\right)\right\}+\sum_{j=1}^{l}\left\{F_{N L, \dot{q}, i, j}\left(\dot{q}_{a}(t)\right)\right\}+ \\
+\sum_{j=1}^{m}\left\{F_{N L, g, i, j}\left(q_{a}(t), \dot{q}_{a}(t)\right)\right\}=\{0\}, \text { with }(k, l, m) \in \mathbb{N}^{*} \times \mathbb{N}^{*} \times \mathbb{N}^{*}(14 \mathrm{c})^{1}
\end{gathered}
$$

whereas the first vector in equation (9), can be decomposed as a sum of $k$-vectors, the second vector of equation (9) can be decomposed as a sum of $l$-vectors, and the last vector of equation (9) as a sum of $m$-vectors, as the final part of equations (14c) is indicating.

a) Neglecting the nonlinear terms in equations (14a-b), leads to the associated linear perpetual mechanical system, and equation (14c) is taking the form,

$$
\left[C_{i, j}\right] \times\{1\} \cdot \dot{q}_{a}(t)+\left[K_{i, j}\right] \times\{1\} \cdot q_{a}(t)=\{0\}, \forall\left(q_{a}, \dot{q}_{a}\right) \in \mathbb{R} \times \mathbb{R}^{*},
$$

\footnotetext{
${ }^{1}$ In case that the proof of the theorem or the corollary given in [21], [22] respectively, is within the document, the proof until this point is redundant.
} 
which is valid for any combination of arbitrary values of generalized coordinates, and of their non-zero velocities leading to,

$$
\left[K_{i, j}\right] \times\{1\} \cdot q_{a}(t)=\{0\}
$$

and,

$$
\left[C_{i, j}\right] \times\{1\} \cdot \dot{q}_{a}(t)=\{0\} .
$$

Taking into account equations $(15 \mathrm{a}, \mathrm{b})$ into equation $(14 \mathrm{c})$ leads to,

$$
\begin{gathered}
\left\{F_{N L, q, i}\left(q_{a}(t)\right)\right\}+\left\{F_{N L, \dot{q}, i}\left(\dot{q}_{a}(t)\right)\right\}+\left\{F_{N L, g, i}\left(q_{a}(t), \dot{q}_{a}(t)\right)\right\}= \\
=\sum_{j=1}^{k}\left\{F_{N L, q, i, j}\left(q_{a}(t)\right)\right\}+\sum_{j=1}^{l}\left\{F_{N L, \dot{q}, i, j}\left(\dot{q}_{a}(t)\right)\right\}+ \\
+\sum_{j=1}^{m}\left\{F_{N L, g, i, j}\left(q_{a}(t), \dot{q}_{a}(t)\right)\right\}=\{0\},
\end{gathered}
$$

In the exact augmented perpetual manifolds all the nonlinear internal force's vectors required to be orthogonal to each other, and multiplying equation (15c) with the transpose of the $r$-element of the first sum of vectors of equation (15c) leads to,

$$
\begin{array}{r}
\left\{F_{N L, q, i, r}\left(q_{a}(t)\right)\right\}^{T} \times\left\{\sum_{j=1}^{k}\left\{F_{N L, q, i, j}\left(q_{a}(t)\right)\right\}+\sum_{j=1}^{l}\left\{F_{N L, \dot{q}, i, j}\left(\dot{q}_{a}(t)\right)\right\}\right. \\
\left.+\sum_{j=1}^{m}\left\{F_{N L, g, i, j}\left(q_{a}(t), \dot{q}_{a}(t)\right)\right\}\right\}=0 \Rightarrow \sum_{i=1}^{N}\left(F_{N L, q, i, r}\left(q_{a}(t)\right)\right)^{2}=0 \Rightarrow \\
\Rightarrow\left\{F_{N L, q, i, r}\left(q_{a}(t)\right)\right\}=\{0\}, \text { with } \quad r \in\{1, \ldots, k\}
\end{array}
$$

Repeating the same pre-multiplication of equation (15c) using the $h$-element of the second sum of vectors of the nonlinear forces of equation (15c) leads to,

$$
\sum_{i=1}^{N}\left(F_{N L, \dot{q}, i, h}\left(\dot{q}_{a}(t)\right)\right)^{2}=0 \Rightarrow\left\{F_{N L, \dot{q}, i, h}\left(\dot{q}_{a}(t)\right)\right\}=\{0\}, \text { with } h \in\{1, \ldots, l\}(1
$$

And with the $s$-element of the third sum of vectors of the nonlinear forces of equation (15c) leads to,

$$
\begin{array}{r}
\sum_{i=1}^{N}\left(F_{N L, g, i, s}\left(q_{a}(t), \dot{q}_{a}(t)\right)\right)^{2}=0 \Longrightarrow\left\{F_{N L, g, i, s}\left(q_{a}(t), \dot{q}_{a}(t)\right)\right\}=\{0\}, \text { with } \\
s \in\{1, \ldots, m\}(15 f)
\end{array}
$$

Therefore in the exact augmented perpetual manifolds, all the individual terms of internal forces vectors, based on the decompositions indicated in equation (14c), they are zero. 
b) Considering the definition of power and equations (15a,15d), then the potential energy in the exact augmented perpetual manifolds is given by,

$$
U_{t o t, a}\left(q_{a}(t)\right)=U_{L}\left(q_{a}(t)\right)+U_{N L}\left(q_{a}(t)\right)=
$$

$=\int_{t_{0}}^{t}\left\{\left[K_{i j}\right] \times\left\{q_{a}(\tau)\right\}\right\}^{T} \times\left\{\dot{q}_{a}(\tau)\right\} d \tau+\int_{t_{0}}^{t}\left\{\left\{F_{N L, q, i}\left(q_{a}(\tau)\right)\right\}\right\}^{T} \times\left\{\dot{q}_{a}(\tau)\right\} d \tau+$

$+U_{t o t, a}\left(t_{0}\right)=\int_{t_{0}}^{t}\{0\}^{T} \times\left\{\dot{q}_{a}(\tau)\right\} d \tau+\int_{t_{0}}^{t}\{0\}^{T} \times\left\{\dot{q}_{a}(\tau)\right\} d \tau+U_{t o t, a}\left(t_{0}\right)=$

$=\frac{1}{2} \cdot\{1\} \times\left[K_{i j}\right] \times\{1\} \cdot q_{a}^{2}\left(t_{0}\right)+U_{N L, a}\left(q_{a}\left(t_{0}\right)\right)=U_{N L, a}\left(q_{a}\left(t_{0}\right)\right),(16$

and remains constant, with it's initial value in the exact augmented perpetual manifolds, that is associated with the nonlinear forces. Therefore during the dynamic evolution there is no energy storage as potential energy on the mechanical system.

c) The energy associated with the velocities' depended linear forces is given by the first term of equation (10c), and in the exact augmented perpetual manifolds considering equation (15b) is taking the form,

$$
\begin{gathered}
E_{L, \dot{q}, a}(t)=\int_{t_{0}}^{t}\left\{\left[C_{i j}\right] \times\left\{\dot{q}_{a}(\tau)\right\}\right\}^{T} \times\left\{\dot{q}_{a}(\tau)\right\} d \tau+E_{L, \dot{q}, a}\left(t_{0}\right)= \\
=\int_{t_{0}}^{t}\{0\}^{T} \times\left\{\dot{q}_{a}(\tau)\right\} d \tau=0 .
\end{gathered}
$$

The energy associated with the velocity depended nonlinear forces, is given by the second term of equation (10c), and in the exact augmented perpetual manifolds considering equation (15e) leads to,

$$
\begin{aligned}
E_{N L, \dot{q}, a}(t) & =\int_{t_{0}}^{t}\left\{F_{N L, \dot{q}, i}\left(\dot{q}_{a}(\tau)\right)\right\}^{T} \times\left\{\dot{q}_{a}(\tau)\right\} d \tau= \\
& =\int_{t_{0}}^{t}\{0\}^{T} \times\left\{\dot{q}_{a}(\tau)\right\} d \tau=0 .
\end{aligned}
$$

The energy associated with the generalized force is given by equation (10d), and considering equation (15f) that is valid in the exact augmented perpetual manifolds, then is taking the form,

$$
\begin{gathered}
E_{N L, g, a}(t)=\int_{t_{0}}^{t}\left\{F_{N L, g, i}\left(q_{a}(\tau), \dot{q}_{a}(\tau)\right)\right\}^{T} \times\left\{\dot{q}_{a}(\tau)\right\} d \tau+ \\
+E_{N L, g, a}\left(t_{0}\right)=\int_{t_{0}}^{t}\{0\}^{T} \times\left\{\dot{q}_{a}(\tau)\right\} d \tau=0 .
\end{gathered}
$$

Therefore, in the exact augmented perpetual manifolds, all the individual types of energies associated with each internal force, are either zero or constant with the initial value. Therefore there is no energy loss as heat due to dissipation, which lead that the internal entropy is, 


$$
\Delta S_{\text {int }}=\frac{\Delta Q}{T}=0
$$

and the process in the exact augmented perpetual manifolds is isentropic [23].

d) In the augmented perpetual manifold, considering the overall system that the external forces are provided to the perpetual mechanical system through an adiabatic process then,

$$
\Delta S_{\text {ext }}=\frac{\Delta Q}{T}=0,
$$

and by adding equation (20a) leads to,

$$
\Delta S=\frac{\Delta Q}{T}=0,
$$

therefore, the perpetual mechanical system with the surroundings is behaving as a perpetual machine of $2^{\text {nd }}$ kind [23,25-26].

e) Rearranging the characteristic differential equation (6), multiplying it with the velocity of the associated generalized velocity $-\dot{q}_{a}$, and then integrate in time lead to,

$$
\begin{gathered}
\sum_{j=1}^{N} M_{k, j} \cdot \int_{t_{0}}^{t} \ddot{q}_{a}(\tau) \cdot \dot{q}_{a}(\tau) d \tau=\int_{t_{0}}^{t} F_{k}\left(\tau, q_{a}(\tau), \dot{q}_{a}(\tau)\right) \cdot \dot{q}_{a}(\tau) d \tau= \\
=W_{e x, a, k}(t) .
\end{gathered}
$$

In the middle of equation (22), the terms inside the integral correspond to the power associated with the $k$-external force $\left(F_{k}\right)$ and therefore, the function arising with integration is the work $\left(W_{e x, a, k}\right)$ that is done by the $k$-external force $\left(F_{k}\right)$ and it is the energy provided to or removed from the system. The integral of equation (22) exists for smooth characteristic differential equations of the augmented perpetual manifolds. In the case of a nonsmooth characteristic differential equation, herein must form a Filippov's system. In Filippov's systems, the velocities are smooth, and these integrals, integration for locally bounded functions of external forces at discontinuities, can be determined with Lebesgue-Stieltjes [34]. The notation at discontinuity, occurring at the time instant $t_{d i s} \in\left[t_{d i s}^{-}, t_{d i s}^{+}\right]$, is a superscript with negative sign (-) indicating the time instant just before the discontinuity and a superscript with positive sign (+) just after the discontinuity. Examining the locally bounded variation of the power of the external force at a discontinuity, the vector fields since is forming a 
Filippov's system have locally bounded variation at discontinuities, therefore,

$$
\begin{gathered}
\operatorname{var}\left(F_{k}\left(\tau, q_{a}(\tau), \dot{q}_{a}(\tau)\right) \cdot \dot{q}_{a}(\tau),\left[t_{d i s}^{-}, t_{d i s}^{+}\right]\right)= \\
=\sup \| F_{k}^{+}\left(t_{d i s}^{+}, q_{a}\left(t_{d i s}^{+}\right), \dot{q}_{a}\left(t_{d i s}^{+}\right)\right) \cdot \dot{q}_{a}\left(t_{d i s}\right)- \\
-F_{k}^{-}\left(t_{d i s}^{-}, q_{a}\left(t_{d i s}^{-}\right), \dot{q}_{a}\left(t_{d i s}^{-}\right)\right) \cdot \dot{q}_{a}\left(t_{d i s}^{-}\right) \|= \\
=\sup \left(\left|\dot{q}_{a}\left(t_{d i s}\right)\right| \cdot\right. \\
\left\|F_{k}^{+}\left(t_{d i s}^{+}, q_{a}\left(t_{d i s}\right), \dot{q}_{a}\left(t_{d i s}\right)\right)-F_{k}^{-}\left(t_{d i s}^{-}, q_{a}\left(t_{d i s}\right), \dot{q}_{a}\left(t_{d i s}\right)\right)\right\|<\infty, \\
\quad \text { for }\left|\dot{q}_{a}\left(t_{d i s}\right)\right|<\infty,
\end{gathered}
$$

The integration by parts of the left-hand side of equation (22) using Riemman's integral requires that the velocity and the acceleration must be continuous functions. In the case of non-smooth systems, to simplify this proof, at the discontinuities, the $\mathrm{Y}$-integral additional terms are considered, that is given in the '19.3.13 theorem for integration by parts' in [35]. The theorem's validity requires that the integrand functions $(f, g)$ must have bounded variation in discontinuities, but in this case the integrands $f=g=\dot{q}_{a}$ are continuous functions, therefore the existence of acceleration discontinuities leads that in the integration by parts, the $\mathrm{Y}$ integral additional terms are zero. Therefore the same formula given by the Reimann integral is applied and leads to,

$$
\int_{t_{0}}^{t} \ddot{q}_{a}(\tau) \cdot \dot{q}_{a}(\tau) d \tau=\frac{1}{2} \cdot\left(\dot{q}_{a}^{2}(t)-\dot{q}_{a}^{2}\left(t_{0}\right)\right) .
$$

Therefore, taking into account equation (24) into equation (22) lead to,

$$
\begin{gathered}
\frac{1}{2} \cdot \sum_{j=1}^{N} M_{k, j} \cdot\left(\dot{q}_{a}^{2}(t)-\dot{q}_{a}^{2}\left(t_{0}\right)\right)= \\
=\int_{t_{0}}^{t} F_{k}\left(\tau, q_{a}(\tau), \dot{q}_{a}(\tau)\right) \cdot \dot{q}_{a}(\tau) d \tau=W_{e x, a, k}(t)=\frac{\sum_{j=1}^{N} M_{k, j}}{\sum_{i=1}^{N} \sum_{j=1}^{N} M_{i, j}} \cdot W_{e x, a}(t),
\end{gathered}
$$

whereas, the last term arise with the use of equation (11c).

Rearrangement of equation (25) lead to,

$$
\begin{gathered}
W_{e x, a}(t)+\frac{1}{2} \cdot \sum_{i=1}^{N} \sum_{j=1}^{N} M_{i, j} \cdot \dot{q}_{a}^{2}\left(t_{0}\right)= \\
=\frac{1}{2} \cdot \sum_{i=1}^{N} \sum_{j=1}^{N} M_{i, j} \cdot \dot{q}_{a}^{2}(t) .
\end{gathered}
$$

Also, the $T_{2}$-kinetic energy $\left(E_{T_{2}, a}\right)$ of the system is given by,

$$
E_{T_{2}, a}\left(\dot{q}_{a}(t)\right)=\frac{1}{2} \cdot\left\{\dot{q}_{a}(t)\right\}^{T} \times\left[M_{i j}\right] \times\left\{\dot{q}_{a}(t)\right\}=\frac{1}{2} \cdot\{1\}^{T} \times\left[M_{i j}\right] \times\{1\} \cdot \dot{q}_{a}^{2}(t)=
$$




$$
=\frac{1}{2} \cdot \sum_{i=1}^{N} \sum_{j=1}^{N} M_{i, j} \cdot \dot{q}_{a}^{2}(t)=W_{e x, a}(t)+E_{T, a}\left(\dot{q}_{a}\left(t_{0}\right)\right) .
$$

Therefore, the total work $\left(W_{e x, a}\right)$ done by the external forcing is equal to the change of the kinetic energy $\left(E_{T, a}\right)$ from the system's initial state. When the work done from the external force increases, the same amount of energy is given in the system, and becomes kinetic energy $\left(E_{T, a}\right)$. In case that the work $\left(W_{e x, a}\right)$ done from the external force is decreasing then the system's kinetic energy decreases with the same amount, and there is mechanical energy loss of the system outside to the 'environment' equal to the work done by the external forces.

f) The provided power of the external forces to the subsystem is given by the equation (11b), and, since the inertia coefficients are positive, the power is positive in the time interval that the external force and the velocity are having the same sign or otherwise stated that the two quantities are codirected. Also, the power is the derivative of the work done by the external force in time, and, in the time interval of positive power, then, the work done by the external force is increasing and therefore also the kinetic energy is increasing with the same amount of energy which means that the subsystem behaves as a perpetual machine of $3^{\text {rd }}$ kind [25]. During the time interval that the power of the external forces is negative, the kinetic energy is decreasing therefore the system is losing energy in some form and therefore cannot be considered that behaves as a perpetual machine of a $3^{\text {rd }}$ kind.

\section{Discussion about the conditions:}

Due to the arbitrary form of the nonlinear forces, to avoid in the rigid body motions solutions, with cancelling out of the internal forces by each other, that might lead to the internal transfer of energy, certain conditions are imposed. The condition's necessity herein is explained.

On each equation of the system (13), admitting rigid body motion that the terms of the nonlinear forces' are non-zero, their functional form can be in three forms. The first one is constants (non-zero) that might be cancelled out with each other, 2) linearly dependent on the generalized coordinates and/or velocities that might be cancelled out with the linear forces, and c) nonlinear terms that might be canceled out by each other. The orthogonality condition for the three terms of equation (9) is imposed to avoid the cancelling out of the nonlinear vectors by 
each other, accompanied by the necessity that their sum must be equal to zero (perpetual mechanical system). Initially the case that each vector of equation (9) is having 'single' elements and not comprised by any sum vectors is examined.

In the following example, the associated linear system is a perpetual one, with the following form of nonlinear forces,

$$
\begin{gathered}
{\left[\begin{array}{cc}
m_{1} & 0 \\
0 & m_{2}
\end{array}\right] \times\left\{\begin{array}{l}
\ddot{q}_{1} \\
\ddot{q}_{2}
\end{array}\right\}+\left[\begin{array}{cc}
k_{e, 1} & -k_{e, 1} \\
-k_{e, 1} & k_{e, 1}
\end{array}\right] \times\left\{\begin{array}{l}
q_{1} \\
q_{2}
\end{array}\right\}+} \\
+\left[\begin{array}{cc}
c_{e, 1} & -c_{e, 1} \\
-c_{e, 1} & c_{e, 1}
\end{array}\right] \times\left\{\begin{array}{l}
\dot{q}_{1} \\
\dot{q}_{2}
\end{array}\right\}+ \\
+\left\{\begin{array}{l}
k_{e, 2} \cdot \sin \left(q_{1}-q_{2}+\pi / 2\right)+k_{e, 2} \cdot \sin \left(q_{1}-q_{2}-\pi / 2\right) \\
c_{e, 2} \cdot \sin \left(\dot{q}_{2}-\dot{q}_{1}-\pi / 2\right)+c_{e, 2} \cdot \sin \left(\dot{q}_{2}-\dot{q}_{1}+\pi / 2\right)
\end{array}\right\}= \\
=\left\{\begin{array}{l}
F_{\text {ext }, 1}\left(t, q_{1}, q_{2}, \dot{q}_{1}, \dot{q}_{2}\right) \\
F_{\text {ext }, 2}\left(t, q_{1}, q_{2}, \dot{q}_{1}, \dot{q}_{2}\right)
\end{array}\right\},
\end{gathered}
$$

whereas each force's vector with linear elements, in rigid body motions, is equal to zero. In the exact augmented perpetual manifolds the first two nonlinear forces are not necessarily zero but they are given by,

$$
\left\{F_{N L, q, i}\left(q_{a}(\tau)\right)\right\}=\left\{\begin{array}{c}
k_{e, 2} \cdot \sin \left(q_{a}-q_{a}+\pi / 2\right) \\
c_{e, 2} \cdot \sin \left(\dot{q}_{a}-\dot{q}_{a}-\pi / 2\right)
\end{array}\right\}=\left\{\begin{array}{c}
k_{e, 2} \\
-c_{e, 2}
\end{array}\right\},
$$

and,

$$
\left\{F_{N L, \dot{q}, i}\left(\dot{q}_{a}(\tau)\right)\right\}=\left\{\begin{array}{c}
k_{e, 2} \cdot \sin \left(q_{a}-q_{a}-\pi / 2\right) \\
c_{e, 2} \cdot \sin \left(\dot{q}_{a}-\dot{q}_{a}+\pi / 2\right)
\end{array}\right\}=\left\{\begin{array}{c}
-k_{e, 2} \\
c_{e, 2}
\end{array}\right\},
$$

summing them up, it certifies that the mechanical system is perpetual, but the energy is transferred from the elastic potential of the nonlinear forces to another form. The orthogonality conditions are not fulfilled, and this case in the theorem is excluded through the imposed orthogonality conditions.

The $2^{\text {nd }}$ case that the nonlinear forces in rigid body motions might lead to cancelling out of them by the linear forces in the next example is shown. As a second example, the following system is considered,

$$
\begin{gathered}
{\left[\begin{array}{cc}
m_{1} & 0 \\
0 & m_{2}
\end{array}\right] \times\left\{\begin{array}{l}
\ddot{q}_{1} \\
\ddot{q}_{2}
\end{array}\right\}+\left[\begin{array}{cc}
k_{e, 1}+k_{e, 2} & -k_{e, 1} \\
-k_{e, 1} & k_{e, 1}-k_{e, 2}
\end{array}\right] \times\left\{\begin{array}{l}
q_{1} \\
q_{2}
\end{array}\right\}+} \\
+\left[\begin{array}{cc}
c_{e, 1}-c_{e, 2} & -c_{e, 1} \\
-c_{e, 1} & c_{e, 1}-c_{e, 2}
\end{array}\right] \times\left\{\begin{array}{l}
\dot{q}_{1} \\
\dot{q}_{2}
\end{array}\right\}+ \\
+\left\{\begin{array}{l}
k_{e, 2} \cdot \sin \left(q_{1}-q_{2}-\pi / 2\right) \cdot q_{1}+c_{e, 2} \cdot \sin \left(\dot{q}_{1}-\dot{q}_{2}+\pi / 2\right) \cdot \dot{q}_{1} \\
k_{e, 2} \cdot \sin \left(q_{2}-q_{1}+\pi / 2\right) \cdot q_{2}+c_{e, 2} \cdot \sin \left(\dot{q}_{2}-\dot{q}_{1}+\pi / 2\right) \cdot \dot{q}_{2}
\end{array}\right\}= \\
=\left\{\begin{array}{l}
F_{\text {ext }, 1}\left(t, q_{1}, q_{2}, \dot{q}_{1}, \dot{q}_{2}\right) \\
F_{\text {ext }, 2}\left(t, q_{1}, q_{2}, \dot{q}_{1}, \dot{q}_{2}\right)
\end{array}\right\},
\end{gathered}
$$


whereas the negative sign of the $c_{e, 2}$ parameter in the diagonal terms of the matrix multiplying the velocities indicates flutter. In the case of rigid body motions, the internal forces are taking the form,

$$
\begin{gathered}
{\left[\begin{array}{cc}
k_{e, 1}+k_{e, 2} & -k_{e, 1} \\
-k_{e, 1} & k_{e, 1}-k_{e, 2}
\end{array}\right] \times\left\{\begin{array}{l}
q_{a} \\
q_{a}
\end{array}\right\}=\left\{\begin{array}{c}
k_{e, 2} \cdot q_{a} \\
-k_{e, 2} \cdot q_{a}
\end{array}\right\},} \\
{\left[\begin{array}{cc}
c_{e, 1}-c_{e, 2} & -c_{e, 1} \\
-c_{e, 1} & c_{e, 1}-c_{e, 2}
\end{array}\right] \times\left\{\begin{array}{l}
\dot{q}_{a} \\
\dot{q}_{a}
\end{array}\right\}=\left\{\begin{array}{l}
-c_{e, 2} \cdot \dot{q}_{a} \\
-c_{e, 2} \cdot \dot{q}_{a}
\end{array}\right\},} \\
\left\{\begin{array}{l}
k_{e, 2} \cdot \sin \left(q_{a}-q_{a}-\pi / 2\right) \cdot q_{a} \\
k_{e, 2} \cdot \sin \left(q_{a}-q_{a}+\pi / 2\right) \cdot q_{a}
\end{array}\right\}=\left\{\begin{array}{c}
-k_{e, 2} \cdot q_{a} \\
k_{e, 2} \cdot q_{a}
\end{array}\right\}, \\
\left\{\begin{array}{l}
c_{e, 2} \cdot \sin \left(\dot{q}_{a}-\dot{q}_{a}+\pi / 2\right) \cdot \dot{q}_{a} \\
c_{e, 2} \cdot \sin \left(\dot{q}_{a}-\dot{q}_{a}+\pi / 2\right) \cdot \dot{q}_{a}
\end{array}\right\}=\left\{\begin{array}{l}
c_{e, 2} \cdot \dot{q}_{a} \\
c_{e, 2} \cdot \dot{q}_{a}
\end{array}\right\} .
\end{gathered}
$$

Summing equations (28e-h) up lead to zero-sum of the internal forces, this means that the system is perpetual. Noting that the two vectors associated with the nonlinear forces in the case of rigid body motions are orthogonal to each other,

$$
\left[\begin{array}{ll}
-k_{e, 2} \cdot q_{a} & k_{e, 2} \cdot q_{a}
\end{array}\right] \times\left\{\begin{array}{l}
c_{e, 2} \cdot \dot{q}_{a} \\
c_{e, 2} \cdot \dot{q}_{a}
\end{array}\right\}=0
$$

Therefore whereas the nonlinear forces for rigid body motion are linearly dependent on the generalized coordinates and/or velocities, they might be cancelled by the linear forces. Setting up a requirement that the underlying linear system must be perpetual, there is no such chance, and then the theorem is valid. Moreover the aforementioned can be extended easily in the case that each vector with nonlinear forces of equation (9) is comprised of a sum. Then each term might cancel out each other of the same vector e.g. softening and stiffening nonlinearities within the vector with generalized coordinates depended forces, but imposing the orthogonality condition to be valid for each element of each vector of equation (9) there is not such a chance.

\section{Remarks:}

1. In mechanical systems, the velocity is always finite, but the requirement is included for any further application of this formalism.

2. In case that the external forces are zero, then there is no work done, and therefore, the system cannot be considered as a perpetual machine.

3. In the statement of the $2^{\text {nd }}$ law of thermodynamics, the change of a system's entropy is zero only in the case of a reversible process [23]. Following the above theorem, in a motion of a perpetual mechanical system described by the exact augmented perpetual manifolds, the change of the internal entropy is zero irrespective of the reversibility of the 
dynamics and also the existence of an irreversible adiabatic process that can provide the external forces. Elaborating further on, a reversible process of a system occurs, when the system is in one state with evolution either backwards or forward the system in both cases could be in the same state, therefore the states corresponds to limit cycles, or periodic motions. The exact augmented perpetual manifolds of a mechanical system can be obtained with various forms of external forces that are not necessarily periodic solutions, e.g. the $1^{\text {st }}$ type of external forces that the solution in the exact augmented perpetual manifolds as in [21] is determined do not periodic motion. In the next section the same external force, without periodic solution, is applied, to examine the individual terms of energies of the system. Therefore the reversibility is not a requirement for constant entropy of the system throughout the dynamics. Subsequently the 'arrow of time' is not necessarily associated with the entropy of the mechanical subsystem.

4. Recalling in the exact augmented perpetual manifolds for all time instants the $2 \cdot N+1$ state space is formed by the time variable and the repetition of the generalized coordinates and velocities, which are the same with the centre of the mass. In another view, the microstates are coinciding with the macrostates of the system. The examination of the perpetual mechanical systems in the exact augmented perpetual manifolds, with the view of statistical mechanics, leads to an interesting ensemble, whereas all the microstates coinciding with the macrostates of the system. This study is out of the scope of this work and can be done elsewhere, including verifying the isentropic character of the process from the statistical mechanics perspective [23].

A natural mechanical system behaves as a perpetual machine based on the combination of the 'environment' and the mechanical system configuration itself (perpetual).

In the augmented perpetual manifolds the input energy to the system is taking the form,

$$
\begin{aligned}
E_{\text {in }, a}(t) & =W_{e x, a}(t)+E_{T}\left(q_{a}\left(t_{0}\right), \dot{q}_{a}\left(t_{0}\right)\right)+U_{N L}\left(q_{a}\left(t_{0}\right)\right)= \\
& =E_{T}\left(q_{a}(\mathrm{t}), \dot{q}_{a}(t)\right)+U_{N L}\left(q_{a}\left(t_{0}\right)\right)=E_{\text {mech }}(t),
\end{aligned}
$$


whereas in case of zero initial potential energy associated with the nonlinear forces, the input energy is equal to the kinetic energy and also to the mechanical energy to the system.

\subsection{Corollary}

After the proof of the theorem of the previous section, a corollary herein is stated and proved.

\section{Corollary}

A perpetual natural mechanical discrete system, in reversible dynamics, and solution in the exact augmented perpetual manifolds, although each individual internal forces cannot behave for the total time interval as a perpetual machine of 3rd kind.

Proof:

In case that the system is starting from a given position and a velocity that either increase (decrease) considering periodicity, the system must come back to the original state with the initial velocity, therefore at a certain time instant the velocity must decrease (increase) which means that for certain time intervals the velocity increase and some others of the same cycle decrease. Since the velocity is straightforwardly correlated with the kinetic energy that in the exact augmented perpetual manifolds for zero initial potential energy is equal to the mechanical energy this means the perpetual mechanical system in certain time intervals is losing energy (in any form that is associated with the work done by the external forces) and therefore on these time intervals cannot behave as a perpetual machine of $3^{\text {rd }}$ kind. Also, if the external forces are zero, the velocities are constant, and then there is no work transferred to the mechanical system, and it cannot be considered as a perpetual machine of $3^{\text {rd }}$ kind.

There are many books in thermodynamics discussing possible ideal ways that the $2^{\text {nd }}$ thermodynamics law is valid, and the 'ideal' perpetual machines. This corollary gives more insight in dynamical processes of mechanical systems that behave as perpetual machines. 


\subsection{Analytical results}

\subsubsection{Analytical examination of energies}

In [21], for two types of external forces, the explicit form of the analytical solutions in the exact augmented perpetual manifolds are derived, and in Table A.1 of App. A is shown.

In this section, the explicit form of the kinetic energy $\left(E_{T, a}^{(r)}\right)$, of the external forces power $\left(P_{e x, a}^{(r)}\right)$ and of their work $\left(E_{e x, a}^{(r)}\right)$ done, in exact augmented perpetual manifolds solutions, associated with each one of these two types of external forces $\left(F_{k}^{(r)}\right)$ given in Table A.1 are determined.

Considering equation (11a) that defines the kinetic energy for a solution of a mechanical system in the exact augmented perpetual manifolds, then -The kinetic energy $\left(E_{T, a}^{(1)}\right)$ associated with a velocity given by the equation (A.2a) for the $1^{\text {st }}$ type of external forces $\left(F_{k}^{(1)}(t)\right)$ in Table A.1, is given by,

$$
\begin{gathered}
E_{T, a}^{(1)}(t)=\frac{1}{2} \cdot \sum_{i=1}^{N} \sum_{j=1}^{N} M_{i, j} \cdot \dot{x}_{a, 1}^{2}(t)=\frac{1}{2} \cdot \sum_{i=1}^{N} \sum_{j=1}^{N} M_{i, j} \cdot\left(\frac{\eta}{2 \cdot \sum_{j=1}^{N} M_{k, j}} \cdot t^{2}+\right. \\
\left.+\frac{c}{\sum_{j=1}^{N} M_{k, j}} \cdot t-\frac{\eta}{2 \cdot \sum_{j=1}^{N} M_{k, j}} \cdot t_{0}^{2}-\frac{c}{\sum_{j=1}^{N} M_{k, j}} \cdot t_{0}+\dot{x}_{a, 1}\left(t_{0}\right)\right)^{2}= \\
=\frac{1}{2} \cdot \sum_{i=1}^{N} \sum_{j=1}^{N} M_{i, j} \cdot\left(\frac{\eta^{2}}{4 \cdot\left(\sum_{j=1}^{N} M_{k, j}\right)^{2}} \cdot t^{4}+\frac{\eta \cdot c}{\left(\sum_{j=1}^{N} M_{k, j}\right)^{2}} \cdot t^{3}-\right. \\
-\left(\frac{\eta^{2} \cdot t_{0}^{2}}{2 \cdot\left(\sum_{j=1}^{N} M_{k, j}\right)^{2}}+\frac{\eta \cdot c \cdot t_{0}}{\left(\sum_{j=1}^{N} M_{k, j}\right)^{2}}-\frac{c^{2}}{\left(\sum_{j=1}^{N} M_{k, j}\right)^{2}}-\frac{\eta \cdot \dot{x}_{a, 1}\left(t_{0}\right)}{\sum_{j=1}^{N} M_{k, j}}\right) \cdot t^{2}- \\
-\left(\frac{\eta \cdot c \cdot t_{0}^{2}}{\left(\sum_{j=1}^{N} M_{k, j}\right)^{2}}+\frac{2 \cdot c^{2} \cdot t_{0}}{\left(\sum_{j=1}^{N} M_{k, j}\right)^{2}}-\frac{2 \cdot c \cdot \dot{x}_{a, 1}\left(t_{0}\right)}{\sum_{j=1}^{N} M_{k, j}}\right) \cdot t+\frac{\eta^{2} \cdot t_{0}^{4}}{4 \cdot\left(\sum_{j=1}^{N} M_{k, j}\right)^{2}}+ \\
\left.+\frac{c^{2} \cdot t_{0}^{2}}{\left(\sum_{j=1}^{N} M_{k, j}\right)^{2}}+\frac{\eta \cdot c \cdot t_{0}^{3}}{\left(\sum_{j=1}^{N} M_{k, j}\right)^{2}}-\frac{\eta \cdot \dot{x}_{a, 1}\left(t_{0}\right) \cdot t_{0}^{2}}{\sum_{j=1}^{N} M_{k, j}}-\frac{2 \cdot c \cdot \dot{x}_{a, 1}\left(t_{0}\right) \cdot t_{0}}{\sum_{j=1}^{N} M_{k, j}}+\dot{x}_{a, 1}^{2}\left(t_{0}\right)\right) \cdot(30
\end{gathered}
$$

-The kinetic energy $\left(E_{T, a}^{(2)}\right)$ associated with a velocity given by the equation (A.2b) for the $2^{\text {nd }}$ type of external forces $\left(F_{k}^{(2)}(t)\right)$ in Table A.1, is given by, 


$$
\begin{gathered}
E_{T, a}^{(2)}(t)=\frac{1}{2} \cdot \sum_{i=1}^{N} \sum_{j=1}^{N} M_{i, j} \cdot \dot{x}_{a, 2}^{2}(t)=\frac{1}{2} \cdot \sum_{i=1}^{N} \sum_{j=1}^{N} M_{i, j} \cdot \\
\cdot\left(-\frac{A_{e x} \cdot \cos \left(\omega_{e x} \cdot t+\theta_{e x}\right)}{\omega_{e x} \cdot \sum_{j=1}^{N} M_{k, j}}+\frac{A_{e x} \cdot \cos \left(\omega_{e x} \cdot t_{0}+\theta_{e x}\right)}{\omega_{e x} \cdot \sum_{j=1}^{N} M_{k, j}}+\dot{x}_{a, 2}\left(t_{0}\right)\right)^{2}= \\
=\frac{1}{2} \cdot \sum_{i=1}^{N} \sum_{j=1}^{N} M_{i, j} \cdot\left(\frac{A_{e x}^{2}}{\left(\sum_{j=1}^{N} M_{k, j}\right)^{2} \cdot \omega_{e x}^{2}} \cdot \cos ^{2}\left(\omega_{e x} \cdot t+\theta_{e x}\right)-\right. \\
-\frac{2 \cdot A_{e x} \cdot \dot{x}_{a, 2}\left(t_{0}\right)}{\sum_{j=1}^{N} M_{k, j} \cdot \omega_{e x}} \cdot \cos \left(\omega_{e x} \cdot t+\theta_{e x}\right) \\
-\frac{2 \cdot A_{e x}^{2} \cdot \cos \left(\omega_{e x} \cdot t_{0}+\theta_{e x}\right)}{\left(\sum_{j=1}^{N} M_{k, j}\right)^{2} \cdot \omega_{e x}^{2}} \cdot \cos \left(\omega_{e x} \cdot t+\theta_{e x}\right)+ \\
\left.+\frac{A_{e x}^{2} \cdot \cos ^{2}\left(\omega_{e x} \cdot t_{0}+\theta_{e x}\right)}{\left(\sum_{j=1}^{N} M_{k, j}\right)^{2} \cdot \omega_{e x}^{2}}+\frac{2 \cdot A_{e x} \cdot \cos \left(\omega_{e x} \cdot t_{0}+\theta_{e x}\right) \cdot \dot{x}_{a, 2}\left(t_{0}\right)}{\sum_{j=1}^{N} M_{k, j} \cdot \omega_{e x}}+\dot{x}_{a, 2}^{2}\left(t_{0}\right)\right)
\end{gathered}
$$

The power $\left(P_{e x, a}\right)$ of the external forces in the exact augmented perpetual manifolds is given by equation (11b). The power associated with the two types of the external forces, given in Table A.1, is, -The power $\left(P_{e x, a}^{(1)}\right)$ of all the external forces, associated with the $1^{\text {st }}$ type of external forces $\left(F_{k}^{(1)}(t)\right)$ given by equation (A.1a) in Table A.1, and velocity given by equation (A.2a) in Table A.1, in explicit form is,

$$
\begin{gathered}
P_{e x, a}^{(1)}(t)=\frac{\sum_{i=1}^{N} \sum_{j=1}^{N} M_{i, j}}{\sum_{j=1}^{N} M_{k, j}} \cdot F_{k}^{(1)}(t) \cdot \dot{x}_{a, 1}(t)= \\
=\frac{\sum_{i=1}^{N} \sum_{j=1}^{N} M_{i, j}}{\sum_{j=1}^{N} M_{k, j}} \cdot(\eta \cdot t+c) \cdot \\
\cdot\left(\frac{\eta}{2 \cdot \sum_{j=1}^{N} M_{k, j}} \cdot\left(t^{2}-t_{0}^{2}\right)+\frac{c}{\sum_{j=1}^{N} M_{k, j}} \cdot\left(t-t_{0}\right)+\dot{x}_{a, 1}\left(t_{0}\right)\right)=\frac{\sum_{i=1}^{N} \sum_{j=1}^{N} M_{i, j}}{\sum_{j=1}^{N} M_{k, j}} . \\
\cdot\left(\frac{\eta^{2}}{2 \cdot \sum_{j=1}^{N} M_{k, j}} \cdot\left(t^{2}-t_{0}^{2}\right) \cdot \mathrm{t}+\frac{c^{2}}{\sum_{j=1}^{N} M_{k, j}} \cdot\left(t-t_{0}\right)+\frac{\eta \cdot c}{\sum_{j=1}^{N} M_{k, j}} \cdot\left(t-t_{0}\right) \cdot t+\right. \\
\left.+\frac{\eta \cdot c}{2 \cdot \sum_{j=1}^{N} M_{k, j}} \cdot\left(t^{2}-t_{0}^{2}\right)+\eta \cdot \dot{x}_{a, 1}\left(t_{0}\right) \cdot t+c \cdot \dot{x}_{a, 1}\left(t_{0}\right)\right)
\end{gathered}
$$

-The power $\left(P_{e x, a}^{(2)}\right)$ for the $2^{\text {nd }}$ type of external forces $\left(F_{k}^{(2)}(t)\right)$ that are given by equation (A.1b) in Table A.1, and velocity given by equation (A.2b) in Table A.1, in explicit form is, 


$$
\begin{gathered}
P_{e x, a}^{(2)}(t)=\frac{\sum_{i=1}^{N} \sum_{j=1}^{N} M_{i, j}}{\sum_{j=1}^{N} M_{k, j}} \cdot F_{k}^{(2)} \cdot \dot{x}_{a, 2}(t)= \\
=\frac{\sum_{i=1}^{N} \sum_{j=1}^{N} M_{i, j}}{\sum_{j=1}^{N} M_{k, j}} \cdot A_{e x} \cdot \sin \left(\omega_{e x} \cdot t+\theta_{e x}\right) \cdot \\
\cdot\left(-\frac{A_{e x} \cdot \cos \left(\omega_{e x} \cdot t+\theta_{e x}\right)}{\omega_{e x} \cdot \sum_{j=1}^{N} M_{k, j}}+\frac{A_{e x} \cdot \cos \left(\omega_{e x} \cdot t_{0}+\theta_{e x}\right)}{\omega_{e x} \cdot \sum_{j=1}^{N} M_{k, j}}+\dot{x}_{a, 2}\left(t_{0}\right)\right)= \\
=\sum_{i=1}^{N} \sum_{j=1}^{N} M_{i, j} \cdot\left(-\frac{A_{e x}^{2}}{\left(\sum_{j=1}^{N} M_{k, j}\right)^{2} \cdot \omega_{e x}} \cdot \sin \left(\omega_{e x} \cdot t+\theta_{e x}\right) \cdot \cos \left(\omega_{e x} \cdot t+\theta_{e x}\right)+\right. \\
\left.+\left(\frac{A_{e x}^{2} \cdot \cos \left(\omega_{e x} \cdot t_{0}+\theta_{e x}\right)}{\left(\sum_{j=1}^{N} M_{k, j}\right)^{2} \cdot \omega_{e x}}+\frac{A_{e x} \cdot \dot{x}_{a, 2}\left(t_{0}\right)}{\sum_{j=1}^{N} M_{k, j}}\right) \cdot \sin \left(\omega_{e x} \cdot t+\theta_{e x}\right)\right) .
\end{gathered}
$$

The work done $\left(W_{e x, a}\right)$ by the external forces in the exact augmented perpetual manifolds is given with integration of the external forces power in time and in general form by the equation (11c). The explicit form of the external work done by the two types of the external forces, given in Table A.1, is,

-The work $\left(W_{e x, a}^{(1)}\right)$ done by all the external forces of the $1^{\text {st }}$ type $\left(F_{k}^{(1)}\right)$ defined by equation (A.1a), with direction integration of equation (31a) is obtained as follows,

$$
\begin{gathered}
W_{e x, a}^{(1)}(t)=\frac{\sum_{i=1}^{N} \sum_{j=1}^{N} M_{i, j}}{\sum_{j=1}^{N} M_{k, j}} \cdot \int_{t_{0}}^{t} F_{k}^{(1)}(t) \cdot \dot{x}_{a, 1}(\tau) d \tau= \\
=\frac{\sum_{i=1}^{N} \sum_{j=1}^{N} M_{i, j}}{\sum_{j=1}^{N} M_{k, j}} \cdot \int_{t_{0}}^{t}\left(\frac{\eta^{2}}{2 \cdot \sum_{j=1}^{N} M_{k, j}} \cdot\left(\tau^{2}-t_{0}^{2}\right) \cdot \tau+\frac{c^{2}}{\sum_{j=1}^{N} M_{k, j}} \cdot\left(\tau-t_{0}\right)+\right. \\
+\frac{\eta \cdot c}{\sum_{j=1}^{N} M_{k, j}} \cdot\left(\tau-t_{0}\right) \cdot \tau \frac{\eta \cdot c}{2 \cdot \sum_{j=1}^{N} M_{k, j}} \cdot\left(\tau^{2}-t_{0}^{2}\right)+ \\
+\frac{\left.+\eta \cdot \dot{x}_{a, 1}\left(t_{0}\right) \cdot \tau+c \cdot \dot{x}_{a, 1}\left(t_{0}\right)\right) d \tau=}{\sum_{i=1}^{N} \sum_{j=1}^{N} M_{i, j}} \cdot\left(\frac{\eta^{2}}{2 \cdot \sum_{j=1}^{N} M_{k, j}} \cdot \int_{t_{0}}^{t} \tau^{3} d \tau+\frac{3 \cdot \eta \cdot c}{2 \cdot \sum_{j=1}^{N} M_{k, j}} \cdot \int_{t_{0}}^{t} \tau^{2} d \tau+\right. \\
+\left(\frac{c^{2}}{\sum_{j=1}^{N} M_{k, j}}-\frac{\eta^{2} \cdot t_{0}^{2}}{2 \cdot \sum_{j=1}^{N} M_{k, j}}-\frac{\eta \cdot c \cdot t_{0}}{\sum_{j=1}^{N} M_{k, j}}+\eta \cdot \dot{x}_{a, 1}\left(t_{0}\right)\right) \cdot \int_{t_{0}}^{t} \tau d \tau+ \\
\left.+\left(-\frac{c^{2} \cdot t_{0}}{\sum_{j=1}^{N} M_{k, j}}-\frac{\eta \cdot c \cdot t_{0}^{2}}{2 \cdot \sum_{j=1}^{N} M_{k, j}}+c \cdot \dot{x}_{a, 1}\left(t_{0}\right)\right) \cdot \int_{t_{0}}^{t} d \tau\right)=
\end{gathered}
$$




$$
\begin{gathered}
=\frac{1}{2} \sum_{i=1}^{N} \sum_{j=1}^{N} M_{i, j} \cdot\left(\frac{\eta^{2}}{4 \cdot\left(\sum_{j=1}^{N} M_{k, j}\right)^{2}} \cdot t^{4}+\frac{\eta \cdot c}{\left(\sum_{j=1}^{N} M_{k, j}\right)^{2}} \cdot t^{3}+\right. \\
-\left(\frac{\eta^{2} \cdot t_{0}^{2}}{2 \cdot\left(\sum_{j=1}^{N} M_{k, j}\right)^{2}}+\frac{\eta \cdot c \cdot t_{0}}{\left(\sum_{j=1}^{N} M_{k, j}\right)^{2}}-\frac{c^{2}}{\left(\sum_{j=1}^{N} M_{k, j}\right)^{2}}-\frac{\eta \cdot \dot{x}_{a, 1}\left(t_{0}\right)}{\sum_{j=1}^{N} M_{k, j}}\right) \cdot t^{2}+ \\
-\left(\frac{\eta \cdot c \cdot t_{0}^{2}}{\left(\sum_{j=1}^{N} M_{k, j}\right)^{2}}+\frac{2 \cdot c^{2} \cdot t_{0}}{\left(\sum_{j=1}^{N} M_{k, j}\right)^{2}}-\frac{2 \cdot c \cdot \dot{x}_{a, 1}\left(t_{0}\right)}{\sum_{j=1}^{N} M_{k, j}}\right) \cdot t+ \\
+\frac{\eta^{2} \cdot t_{0}^{4}}{4 \cdot\left(\sum_{j=1}^{N} M_{k, j}\right)^{2}}+\frac{c^{2} \cdot t_{0}^{2}}{\left(\sum_{j=1}^{N} M_{k, j}\right)^{2}}+\frac{\eta \cdot c \cdot t_{0}^{3}}{\left(\sum_{j=1}^{N} M_{k, j}\right)^{2}}+ \\
\left.-\frac{\eta \cdot \dot{x}_{a, 1}\left(t_{0}\right) \cdot t_{0}^{2}}{\sum_{j=1}^{N} M_{k, j}}-\frac{2 \cdot c \cdot \dot{x}_{a, 1}\left(t_{0}\right) \cdot t_{0}}{\sum_{j=1}^{N} M_{k, j}}\right)=T_{a}^{(1)}(t)-T_{a}^{(1)}\left(t_{0}\right) .
\end{gathered}
$$

The last term obtained using equation (30a) for any time instant $-t$ and for the time instant $-t_{0}$ that the motion with the $1^{\text {st }}$ type of external forces is starting, and certifies equation (27) that is the e-part of the theorem.

-The work $\left(W_{e x, a}^{(2)}\right)$ done by all the external forces of the $2^{\text {nd }}$ type $\left(F_{k}^{(2)}\right)$ defined by equation (A.1b), with direction integration of equation ( $31 \mathrm{~b})$ is obtained as follows,

$$
\begin{aligned}
& W_{e x, a}^{(2)}(t)=\frac{\sum_{i=1}^{N} \sum_{j=1}^{N} M_{i, j}}{\sum_{j=1}^{N} M_{k, j}} \cdot \int_{t_{0}}^{t} F_{k}^{(2)}(\tau) \cdot \dot{x}_{a, 2}(\tau) d \tau= \\
& =\frac{\sum_{i=1}^{N} \sum_{j=1}^{N} M_{i, j}}{\sum_{j=1}^{N} M_{k, j}} \cdot\left(-\frac{A_{e x}^{2}}{\sum_{j=1}^{N} M_{k, j} \cdot \omega_{e x}} .\right. \\
& \int_{t_{0}}^{t} \sin \left(\omega_{e x} \cdot t+\theta_{e x}\right) \cdot \cos \left(\omega_{e x} \cdot t+\theta_{e x}\right) d \tau+ \\
& +\left(\frac{A_{e x}^{2} \cos \left(\omega_{e x} \cdot t_{0}+\theta_{e x}\right)}{\sum_{j=1}^{N} M_{k, j} \cdot \omega_{e x}}+A_{e x} \cdot \dot{x}_{a, 2}\left(t_{0}\right)\right) \cdot \int_{t_{0}}^{t} \sin \left(\omega_{e x} \cdot \tau+\theta_{e x}\right) d \tau= \\
& =\frac{\sum_{i=1}^{N} \sum_{j=1}^{N} M_{i, j}}{\sum_{j=1}^{N} M_{k, j}} \cdot\left(-\left.\frac{A_{e x}^{2}}{\sum_{j=1}^{N} M_{k, j} \cdot \omega_{e x}}\left[\frac{\sin ^{2}\left(\omega_{e x} \cdot \tau+\theta_{e x}\right)}{2 \cdot \omega_{e x}}\right]\right|_{\tau=t_{0}} ^{\tau=t}+\right. \\
& \left.+\left.\left(\frac{A_{e x}^{2} \cdot \cos \left(\omega_{e x} \cdot t_{0}+\theta_{e x}\right)}{\sum_{j=1}^{N} M_{k, j} \cdot \omega_{e x}}+A_{e x} \cdot \dot{x}_{a, 2}\left(t_{0}\right)\right)\left[-\frac{\cos \left(\omega_{e x} \cdot \tau+\theta_{e x}\right)}{\omega_{e x}}\right]\right|_{\tau=t_{0}} ^{\tau=t}\right)= \\
& =\frac{1}{2} \cdot \sum_{i=1}^{N} \sum_{j=1}^{N} M_{i, j} \cdot\left(\frac{A_{e x}^{2}}{\left(\sum_{j=1}^{N} M_{k, j}\right)^{2} \cdot \omega_{e x}^{2}} \cdot \cos ^{2}\left(\omega_{e x} \cdot t+\theta_{e x}\right)-\right.
\end{aligned}
$$




$$
\begin{aligned}
& -2 \cdot\left(\frac{A_{e x}^{2} \cdot \cos \left(\omega_{e x} \cdot t_{0}+\theta_{e x}\right)}{\left(\sum_{j=1}^{N} M_{k, j}\right)^{2} \cdot \omega_{e x}^{2}}+\frac{A_{e x} \cdot \dot{x}_{a, 2}\left(t_{0}\right)}{\sum_{j=1}^{N} M_{k, j} \cdot \omega_{e x}}\right) \cdot \cos \left(\omega_{e x} \cdot t+\theta_{e x}\right)+ \\
& \left.+\frac{A_{e x}^{2} \cdot \cos ^{2}\left(\omega_{e x} \cdot t_{0}+\theta_{e x}\right)}{\left(\sum_{j=1}^{N} M_{k, j}\right)^{2} \cdot \omega_{e x}^{2}}+\frac{2 \cdot A_{e x} \cdot \cos \left(\omega_{e x} \cdot t_{0}+\theta_{e x}\right) \cdot \dot{x}_{a, 2}\left(t_{0}\right)}{\sum_{j=1}^{N} M_{k, j} \cdot \omega_{e x}}\right)=T_{a}^{(2)}(t)-T_{a}^{(2)}\left(t_{0}\right) \cdot(3
\end{aligned}
$$

The last term of equation (32b) is obtained using equation (30b) for the two time instants, the time instant $-t_{0}$ that the application of the $2^{\text {nd }}$ type of the external force is starting, and any other time instant- $t$. The equality of the work done by the external forces with the difference of the kinetic energies, certify analytically, equation (27) for the $2^{\text {nd }}$ type of forces, and therefore the part-e of the theorem. In this part of the section,

\subsubsection{Analytical examination of external forcing positive power}

This subsection, presents a brief analysis of the conditions that the power of the two types of external forces, given in Table A.1, is positive. The power in augmented perpetual manifolds is given by equation (11b). Therefore for positive power is sufficient to examine the conditions that the following inequality is true,

$$
F_{k}^{(r)} \cdot \dot{q}_{a, r}(t)>0 \text {. }
$$

I. The power $\left(P_{e x, a}^{(1)}\right)$ associated with the linear time-varying external forces $\left(F_{k}^{(1)}(t)\right)$ of Table A.1, is given by the equation (31a).

Ia. In case of $(\eta, c) \in \mathbb{R}_{>0} \times \mathbb{R}_{>0}$ the external forces are positive, and the velocity is given by,

$\dot{q}_{a, 1}(t)=\left(\frac{\eta}{2 \cdot \sum_{j=1}^{N} M_{k, j}} \cdot\left(t^{2}-t_{0}^{2}\right)+\frac{c}{\sum_{j=1}^{N} M_{k, j}} \cdot\left(t-t_{0}\right)+\dot{q}_{a, 1}\left(t_{0}\right)\right)$,

whereas in the case of positive initial velocity $\left(\dot{q}_{a, 1}\left(t_{0}\right) \in \mathbb{R}_{>0}\right)$, then all terms in equation (34a), in all time instants, are positive, resulting in positive power.

Ib. In case of zero initial velocity with positive external force (positive acceleration), the velocity increases and becomes positive and codirected with the external force which lead to positive power.

Ic. In case of negative initial velocity, then equation (34a) is taking the form,

$$
\dot{q}_{a, 1}(t)=\left(\frac{\eta}{2 \cdot \sum_{j=1}^{N} M_{k, j}} \cdot\left(t^{2}-t_{0}^{2}\right)+\frac{c}{\sum_{j=1}^{N} M_{k, j}} \cdot\left(t-t_{0}\right)-\left|\dot{q}_{a, 1}\left(t_{0}\right)\right|\right)=
$$




$$
\begin{aligned}
& =\frac{|\eta|}{2 \cdot \sum_{j=1}^{N} M_{k, j}} \cdot\left(t+\frac{|c|}{|\eta|}+\sqrt{\frac{c^{2}}{\eta^{2}}+t_{0}^{2}+\frac{2 \cdot|c| \cdot t_{0}}{|\eta|}+\frac{2 \cdot \sum_{j=1}^{N} M_{k, j} \cdot\left|\dot{q}_{a, 1}\left(t_{0}\right)\right|}{|\eta|}}\right) . \\
& \cdot\left(t+\frac{|c|}{|\eta|}-\sqrt{\frac{c^{2}}{\eta^{2}}+t_{0}^{2}+\frac{2 \cdot|c| \cdot t_{0}}{|\eta|}+\frac{2 \cdot \sum_{j=1}^{N} M_{k, j} \cdot\left|\dot{q}_{a, 1}\left(t_{0}\right)\right|}{|\eta|}}\right),
\end{aligned}
$$

whereas the last term can be easily verified that is negative and as long as the time passes (increasing $t$ ) the velocity increase and becomes positive, after the time instant, that the last parenthesis term in equation (34b) becomes positive, and is given by,

$$
t=-\frac{|c|}{|\eta|}+\sqrt{\frac{c^{2}}{\eta^{2}}+t_{0}^{2}+\frac{2 \cdot|c| \cdot t_{0}}{|\eta|}+\frac{2 \cdot \sum_{j=1}^{N} M_{k, j} \cdot\left|\dot{q}_{a, 1}\left(t_{0}\right)\right|}{|\eta|}}
$$

and ever since the velocity is positive resulting in positive power.

Id. In case of $(\eta, c) \in \mathbb{R}_{<0} \times \mathbb{R}_{<0}$, the external forces are negative, and the velocity is given by,

$$
\dot{q}_{a, 1}(t)=\left(-\frac{|\eta|}{2 \cdot \sum_{j=1}^{N} M_{k, j}} \cdot\left(t^{2}-t_{0}^{2}\right)-\frac{|c|}{\sum_{j=1}^{N} M_{k, j}} \cdot\left(t-t_{0}\right)+\dot{q}_{a, 1}\left(t_{0}\right)\right)
$$

whereas in the case of negative initial velocity $\left(\dot{q}_{a, 1}\left(t_{0}\right) \in \mathbb{R}_{<0}\right)$, all terms in equation (35a) are negative, and the velocity is decreasing in all time instants resulting in positive power.

Ie. In zero initial velocity with negative external force (acceleration), the velocity decreases and becomes negative, which means co-directed with force and leads to positive power.

If. In case of positive initial velocity $\left(\dot{q}_{a, 1}\left(t_{0}\right) \in \mathbb{R}_{>0}\right)$ with negative external force (acceleration), then equation (35a) is taking the form,

$$
\begin{gathered}
\dot{q}_{a, 1}(t)=\left(-\frac{|\eta|}{2 \cdot \sum_{j=1}^{N} M_{k, j}} \cdot\left(t^{2}-t_{0}^{2}\right)-\frac{|c|}{\sum_{j=1}^{N} M_{k, j}} \cdot\left(t-t_{0}\right)+\left|\dot{q}_{a, 1}\left(t_{0}\right)\right|\right)= \\
=-\frac{|\eta|}{2 \cdot \sum_{j=1}^{N} M_{k, j}} \cdot\left(t+\frac{|c|}{|\eta|}+\sqrt{\left.\frac{c^{2}}{\eta^{2}}+t_{0}^{2}+\frac{2 \cdot|c| \cdot t_{0}}{|\eta|}+\frac{2 \cdot \sum_{j=1}^{N} M_{k, j} \cdot\left|\dot{q}_{a, 1}\left(t_{0}\right)\right|}{|\eta|}\right)}\right. \\
\cdot\left(t+\frac{|c|}{|\eta|}-\sqrt{\frac{c^{2}}{\eta^{2}}+t_{0}^{2}+\frac{2 \cdot|c| \cdot t_{0}}{|\eta|}+\frac{2 \cdot \sum_{j=1}^{N} M_{k, j}\left|\dot{q}_{a, 1}\left(t_{0}\right)\right|}{|\eta|}}\right),
\end{gathered}
$$

then the velocity is decreasing until the time instant that becomes negative. This time instant occurs when the last parenthesis term in 
equation (35b) becomes positive, which is true for the time instant given by the same equation of the previous case (eq. 34c).

A more complicated, case to examine could be the power when the constants in forcing having opposite sign but such thorough examination of all the conditions that lead to positive power is out of this article's scope.

II. Power examination associated with single frequency harmonic external forces $\left(F_{k}^{(2)}(t)\right)$.

The power $P_{e x, a}^{(2)}(t)$ is given by equation (31b) which can take the form,

$$
\begin{gathered}
P_{e x, a}^{(2)}(t)=\frac{\sum_{i=1}^{N} \sum_{j=1}^{N} M_{i, j}}{\sum_{j=1}^{N} M_{k, j}} \cdot A_{e x} \cdot \sin \left(\omega_{e x} \cdot t+\theta_{e x}\right) \cdot \\
\cdot\left(-\frac{A_{e x}}{\sum_{j=1}^{N} M_{k, j^{\prime}} \omega_{e x}} \cdot \sin \left(\omega_{e x} \cdot t+\theta_{e x}+\pi / 2\right)+\frac{A_{e x}}{\sum_{j=1}^{N} M_{k, j^{*}} \omega_{e x}}+\dot{q}_{a, 2}(0)\right),
\end{gathered}
$$

whereas it is profound that the force's sign is alternating every half of the excitation period $\left(t_{a}=T_{e x} / 2=\pi / \omega_{e x}\right)$. When this occurs, since there is a phase difference in the sinusoidal time-dependent function of the velocity, it wouldn't necessarily occur simultaneously.

Since the general case examination is rather complicated, to simplify things without losing the general perspective, some assumptions are made to examine a certain occasion.

Standing waves motion for $A_{e x} \in \mathbb{R}_{>0}$ is examined. Choosing initial conditions that lead to zero wave velocity then,

$$
\dot{q}_{a, 2}\left(t_{0}\right)=-\frac{A_{e x} \cdot \cos \left(\omega_{e x} \cdot t_{0}+\theta_{e x}\right)}{\sum_{j=1}^{N} M_{k, j} \cdot \omega_{e x}},
$$

and the power is given by,

$$
P_{e x, a}^{(2)}(t)=-\frac{\sum_{i=1}^{N} \sum_{j=1}^{N} M_{i, j}}{2 \cdot\left(\sum_{j=1}^{N} M_{k, j}\right)^{2} \cdot \omega_{e x}} \cdot A_{e x}^{2} \cdot \sin \left(2 \cdot \omega_{e x} \cdot t+2 \cdot \theta_{e x}\right) .
$$

It is profound that the power could be positive or negative based on the phase of the sinusoidal function. For the time instants that the phase is,

$$
\omega_{e x} \cdot t+\theta_{e x} \in\left((2 \cdot \mathrm{k}-1) \cdot \frac{\pi}{2}, \mathrm{k} \cdot \pi\right), \quad \text { with } k \in \mathbb{N},
$$

on these time intervals the power of the external force is positive, and the system behaves as a perpetual machine of $3^{\text {rd }}$ kind. For all the other phases the power is negative or zero. 
In the next section, the validity of the theory in two different mechanical systems with motion in exact augmented perpetual manifolds is examined.

\section{APPLICATION OF THE THEORY IN MECHANICAL SYSTEMS}

\subsection{A perpetual mechanical system, model of a five wagons train}

A train with five wagons modeled as five degrees of freedom mechanical is considered, as in Figure 1 is shown.

The equations of motion are given by,

$$
\left[M_{x}\right] \times\left\{\ddot{x}_{i}(t)\right\}+\left\{F_{L, s t}\right\}+\left\{F_{L, d}\right\}+\left\{F_{N L, x, i}\left(x_{l}(t)\right)\right\}+\left\{F_{N L, \dot{x}, i}\left(\dot{x}_{r}(t)\right)\right\}=\left\{F_{x, i}\right\} .
$$

The mass matrix of the train is defined by,

$$
\left[M_{x}\right]=\left[\begin{array}{ccccc}
m_{1} & 0 & 0 & 0 & 0 \\
0 & m_{2} & 0 & 0 & 0 \\
0 & 0 & m_{3} & 0 & 0 \\
0 & 0 & 0 & m_{4} & 0 \\
0 & 0 & 0 & 0 & m_{5}
\end{array}\right],
$$

and $m_{i}(i=1, \ldots, 5)$ are positive constants.

The linear forces based on stiffness matrix is defined by,

$$
\begin{gathered}
\left\{F_{L, s t}\right\}=\left[K_{x}\right] \times\left\{x_{i}(t)\right\}= \\
=\left[\begin{array}{ccccc}
k_{x, 1} & -k_{x, 1} & 0 & 0 & 0 \\
-k_{x, 1} & k_{x, 1}+k_{x, 2} & -k_{x, 2} & 0 & 0 \\
0 & -k_{x, 2} & k_{x, 2}+k_{x, 3} & -k_{x, 3} & 0 \\
0 & 0 & -k_{x, 3} & k_{x, 3}+k_{x, 4} & -k_{x, 4} \\
0 & 0 & 0 & -k_{x, 4} & k_{x, 4}
\end{array}\right] \times\left\{x_{i}(t)\right\},
\end{gathered}
$$

and the linear forces based on the damping matrix defined using Rayleigh damping as follows,

$$
\begin{gathered}
\left\{F_{L, d}\right\}=\left[C_{x}\right] \times\left\{\dot{x}_{i}(t)\right\}= \\
=\left[\begin{array}{ccccc}
c_{x, 1} & -c_{x, 1} & 0 & 0 & 0 \\
-c_{x, 1} & c_{x, 1}+c_{x, 2} & -c_{x, 2} & 0 & 0 \\
0 & -c_{x, 2} & c_{x, 2}+c_{x, 3} & -c_{x, 3} & 0 \\
0 & 0 & -c_{x, 3} & c_{x, 3}+c_{x, 4} & -c_{x, 4} \\
0 & 0 & 0 & -c_{x, 4} & c_{x, 4}
\end{array}\right] \times\left\{\dot{x}_{i}(t)\right\}= \\
=\left(\alpha \cdot\left[M_{x}\right]+\beta_{1} \cdot\left[K_{x}\right]\right) \times\left\{\dot{x}_{i}(t)\right\},
\end{gathered}
$$

with $\alpha=0$. The nonlinear forces vector associated with an elastic potential of nonlinear forces is given by, 


$$
\begin{gathered}
\left\{F_{N L, x, i}\left(x_{l}(t)\right)\right\}= \\
k_{x, n l, 1} \cdot \sin \left(x_{1}-x_{2}\right) \\
=\left\{\begin{array}{c}
k_{x, n l, 1} \cdot \sin \left(x_{1}-x_{2}\right)-c_{x, n l, 1} \cdot\left(\dot{x}_{1}-\dot{x}_{2}\right)^{3}+k_{x, n l, 2} \cdot\left(x_{2}-x_{3}\right)^{5} \\
-k_{x, n l, 2} \cdot\left(x_{2}-x_{3}\right)^{5}+k_{x, n l, 3} \cdot \sin \left(x_{3}-x_{4}\right) \\
-k_{x, n l, 3} \cdot \sin \left(x_{3}-x_{4}\right)+k_{x, n l, 4} \cdot\left(x_{4}-x_{5}\right)^{7} \\
-k_{x, n l, 4} \cdot\left(x_{4}-x_{5}\right)^{7}
\end{array}\right\},
\end{gathered}
$$

and the nonlinear damping forces vector is given by,

$$
\left\{F_{N L, \dot{x}, i}\left(\dot{x}_{r}(t)\right)\right\}=\left\{\begin{array}{c}
c_{x, n l, 1} \cdot\left(\dot{x}_{1}-\dot{x}_{2}\right)^{3} \\
-c_{x, n l, 1} \cdot\left(\dot{x}_{1}-\dot{x}_{2}\right)^{3}+c_{x, n l, 2} \cdot\left(\dot{x}_{2}-\dot{x}_{3}\right)^{5} \\
-c_{x, n l, 2} \cdot\left(\dot{x}_{2}-\dot{x}_{3}\right)^{5}+c_{x, n l, 3} \cdot\left(\dot{x}_{3}-\dot{x}_{4}\right)^{7} \\
-c_{x, n l, 3} \cdot\left(\dot{x}_{3}-\dot{x}_{4}\right)^{7}+c_{x, n l, 4} \cdot \tanh \left(b \cdot\left(\dot{x}_{4}-\dot{x}_{5}\right)\right) \\
-c_{x, n l, 4} \cdot \tanh \left(b \cdot\left(\dot{x}_{4}-\dot{x}_{5}\right)\right)
\end{array}\right\},
$$

noting that the internal nonlinear force between the $4^{\text {th }}$ and $5^{\text {th }}$ wagon, in equation (40e) is smoothed dry friction force.

The external forcing vector is given by,

$$
\left\{F_{x, i}\right\}^{T}=\left[\begin{array}{lllll}
1 & m_{2} / m_{1} & m_{3} / m_{1} & m_{4} / m_{1} & m_{5} / m_{1}
\end{array}\right] \cdot F_{x, 1} \cdot
$$

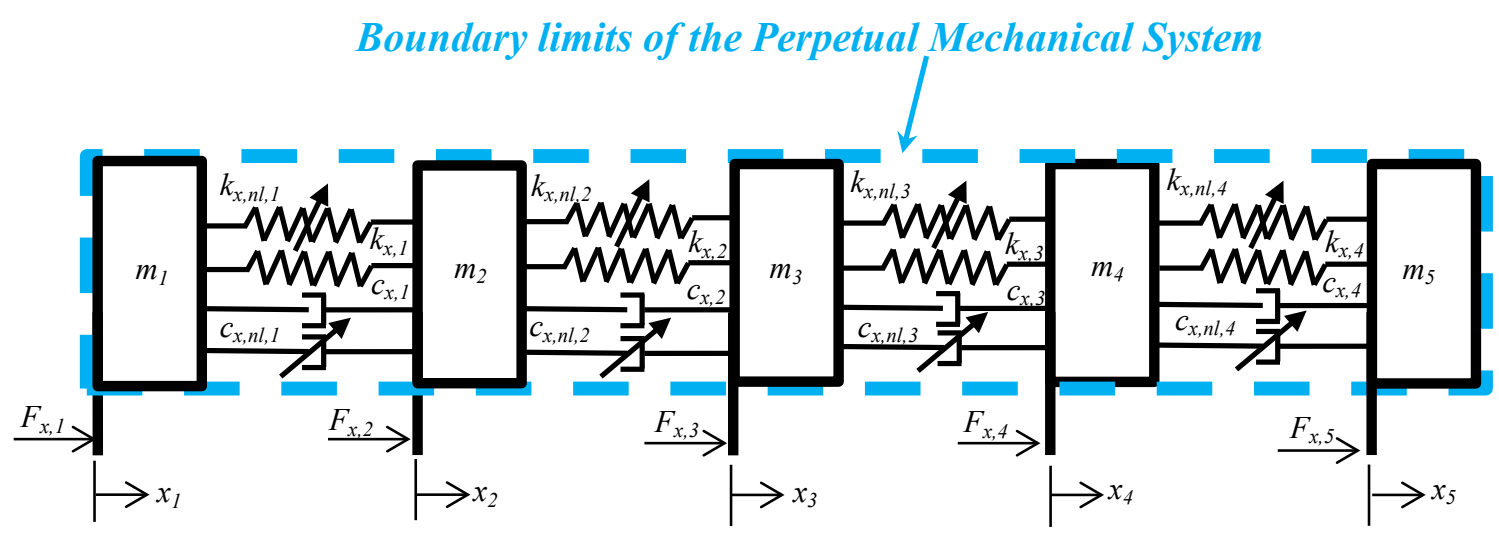

Figure 1. The configuration of the train as a 5-dof mechanical system.

In [21], the same system has been examined, and that it forms a perpetual mechanical system is shown. The same ratio of external forces is used that leads to exact augmented perpetual manifolds solutions, and they are described by the following form of equation (6),

$$
\ddot{x}_{a}(t)=\frac{F_{x, 1}}{m_{1}} .
$$

Since in the exact augmented perpetual manifolds each one internal forcing vector of equation (39) with explicit form of matrices given by the equations (31a-e) is zero, leads that the nonlinear mechanical system is perpetual. Also, the underlying linear system is a perpetual mechanical system, and that the nonlinear forcing 
vectors are all orthogonal to each other, can easily certified. Therefore the conditions of the theorem are fulfilled.

The applied external forces $\left(F_{x, 1}\right)$ for each time interval with the indication of the equations that define the solutions, the wave velocity and the type of motion in Table 1 are shown. It should be highlighted that the system, for the considered total time interval from $0-3 \mathrm{sec}$, since there are various non-convexified discontinuities of the external forces at the beginning of each time-subinterval, the system is not forming a Filippov's system.

The different types of energies used in numerical simulations are given by equations (10) in $\S 2.1$.

-The potential energy terms that are associated with the nonlinear forces in equation (40d) can be given by at least two definitions; either they can be defined by,

$$
\begin{gathered}
U_{N L, 1}(t)=k_{x, n l, 1} \cdot \cos \left(x_{1}(t)-x_{2}(t)\right)+\frac{1}{6} \cdot k_{x, n l, 2} \cdot\left(x_{2}(t)-x_{3}(t)\right)^{6}+ \\
+k_{x, n l, 3} \cdot \cos \left(x_{3}(t)-x_{4}(t)\right)+\frac{1}{8} \cdot k_{x, n l, 4} \cdot\left(x_{4}(t)-x_{5}(t)\right)^{8}
\end{gathered}
$$

or,

$$
\begin{gathered}
U_{N L, 2}(t)=k_{x, n l, 1} \cdot\left(1-\cos \left(x_{1}(t)-x_{2}(t)\right)\right)+\frac{1}{6} \cdot k_{x, n l, 2} \cdot\left(x_{2}(t)-x_{3}(t)\right)^{6}+ \\
\quad+k_{x, n l, 3} \cdot\left(1-\cos \left(x_{3}(t)-x_{4}(t)\right)\right)+\frac{1}{8} \cdot k_{x, n l, 4} \cdot\left(x_{4}(t)-x_{5}(t)\right)^{8} . \quad(42 \mathrm{~b})
\end{gathered}
$$

Both potential functions can be used in the Lagrange equation and lead to the same nonlinear forces given by the equation (40d). The only difference is that in the exact augmented perpetual manifolds, the potential energy given by equations $(42 a, b)$ has different constant referenced value. Starting from equation (42a) and considering exact rigid body motion leads to,

$$
\begin{gathered}
U_{N L, a, 1}(t)=k_{x, n l, 1} \cdot \cos \left(x_{a}(t)-x_{a}(t)\right)+\frac{1}{6} \cdot k_{x, n l, 2} \cdot\left(x_{a}(t)-x_{a}(t)\right)^{6}+ \\
+k_{x, n l, 3} \cdot \cos \left(x_{a}(t)-x_{a}(t)\right)+\frac{1}{8} \cdot k_{x, n l, 4} \cdot\left(x_{a}(t)-x_{a}(t)\right)^{8}= \\
=k_{x, n l, 1}+k_{x, n l, 3} .
\end{gathered}
$$

Considering the potential energy given by equation (42b) in augmented perpetual manifolds, the potential energy is,

$$
\begin{aligned}
& U_{N L, a, 2}(t)=k_{x, n l, 1} \cdot\left(1-\cos \left(x_{a}(t)-x_{a}(t)\right)\right)+\frac{1}{6} \cdot k_{x, n l, 2} \cdot\left(x_{a}(t)-x_{a}(t)\right)^{6}+ \\
& +k_{x, n l, 3} \cdot\left(1-\cos \left(x_{a}(t)-x_{a}(t)\right)\right)+\frac{1}{8} \cdot k_{x, n l, 4} \cdot\left(x_{a}(t)-x_{a}(t)\right)^{8}=0 .
\end{aligned}
$$


In this example, to simplify things, the elastic potential for the nonlinear forces given by equations ( $42 \mathrm{~b}$ ) is used, which lead to zero reference value for the elastic potential in the exact augmented perpetual manifolds. Therefore based on equation (29) in the exact augmented perpetual manifolds, the input energy comprised by the initial kinetic energy adding the work done by the external forces is equal to the kinetic energy; and the mechanical energy of the system. In Table 2, for each time interval, the equations that define the analytical solutions of the kinetic, of the power of the external forces, their work they have done, and the type of system in terms of energy behavior, are shown.

The parameters that define the mass, stiffness, damping and nonlinear forces in Table 3 are shown. The natural frequencies of the underlying linear system obtained by solving the eigenvalue problem in Table 4 are shown, and they are used to determine the damping matrices.

The external forcing parameters and the initial conditions used for each time interval in Table 5 are given. After the first time interval, the initial conditions, on each time interval, arise by the last state from the previous time-interval motion.

\section{Analysis of the system dynamics, for each time interval, in terms of the energy exchange with the environment}

This can be done, based on the type of external forcing (Tables 1, A.1), with their parameters (Table 5), and considering the analysis of the section 2.3.2 about the examination of the sign of the external forces power as follows:

1) For $t \in(0,1]$, the external forcing is $F_{k}^{(1)}$ (Table A.1), and for zero initial velocity, the power is given by the equation (31a). Since the parameters defining the force in Table 4 are positive $\left((\eta, c) \in \mathbb{R}_{>0} \times \mathbb{R}_{>0}\right)$, the analysis (Ia) in subsection 2.4.2, leads to the conclusion that the system behaves as a perpetual machine of $3^{\text {rd }}$ kind.

2) For $t \in(1,2]$, based on the parameters given in Tables 1 and 5, lead that the external force $F_{k}^{(1)}$ is negative. The initial velocity is positive, and as shown in the analysis (If) in subsection 2.4 .2 , with the velocity given by equation (35b), until the moment that is becoming zero, and this time instant is given by equation (34c), the system is losing energy.

Considering the values indicated in Table 5, the velocity is changing sign at the time instant $t=1.4150406 \mathrm{~s}$. Ever since on this time interval the 
velocity is co-directed with the force, resulting in the positive power of the external forcing, and the system behaves as a perpetual machine of $3^{\text {rd }}$ kind.

3) For $t \in(2,3]$, the external forcing is $F_{k}^{(2)}$, and based on analysis (II), the power is having alternated sign. As indicated in Table 3, the wave velocity is zero and therefore, the system returns after the excitation period to the original state. As shown in the analysis (II) of subsection 2.4.2, the system behaves as a perpetual machine of $3^{\text {rd }}$ kind for the time subintervals defined by the equation ( $38 \mathrm{~b}$ ), which is taking the following form,

$$
t \in\left((2 \cdot k-1) \cdot \frac{\pi}{2 \cdot \omega_{e x}}, k \cdot \frac{\pi}{\omega_{e x}}\right), \quad \text { with } k \in \mathbb{N},
$$

the power is positive, and the system behaves as a perpetual machine of $3^{\text {rd }}$ kind, and they are indicated in Table 8 . Therefore, the system is not behaving as a perpetual machine of $3^{\text {rd }}$ kind in all time instants, which certifies the corollary.

\begin{tabular}{|c|c|c|c|c|c|c|}
\hline $\mathrm{i}$ & $\begin{array}{c}\text { Time } \\
\text { interval } \\
\text { (s) }\end{array}$ & $F_{x, 1}^{(i)}$ & $\begin{array}{c}\text { Analytical } \\
\text { velocity } \\
\dot{x}_{a, i}(t)\end{array}$ & $\begin{array}{c}\text { Analytical } \\
\text { displacement } \\
x_{a, i}(t)\end{array}$ & $\begin{array}{c}\text { wave } \\
\text { velocity } \\
w v_{x, i}(t)\end{array}$ & Type of motion \\
\hline 1 & $t \in(0,1]$ & $F_{k}^{(1)}=\eta_{1} \cdot t+c_{1}$ & Eq. (A.2a) & Eq. (A.3a) & - & Particle-curvilinear \\
\hline 2 & $t \in(1,2]$ & $F_{k}^{(1)}=\eta_{2} \cdot t+c_{2}$ & Eq. (A.2a) & Eq. (A.3a) & - & Particle-curvilinear \\
\hline 3 & $t \in(2,3]$ & $F_{k}^{(2)}=A_{e x} \cdot \sin \left(\omega_{e x} \cdot t+\theta_{e x}\right)$ & Eq. (A.2b) & Eq. (A.3b) & Eq. (A.4) & $\begin{array}{l}\text { Particle-standing } \\
\text { wave }\end{array}$ \\
\hline
\end{tabular}

Table 1.The several types of the external forces applied to the train on each time interval, the type of motion, and referencing the explicit form of equations defining the analytical solutions.

\begin{tabular}{|l|l|l|l|l|l|}
\hline \multicolumn{1}{|l|}{} & $\begin{array}{l}\text { Time } \\
\text { interval } \\
(\mathrm{s})\end{array}$ & $\begin{array}{l}\text { Kinetic } \\
\text { Energy } \\
\left.E_{T, a}^{(r)}(t)\right)\end{array}$ & $\begin{array}{l}\text { Power of } \\
\text { external forces } \\
P_{e x, a}^{(r)}(t)\end{array}$ & $\begin{array}{l}\text { Work done } \\
\text { by the } \\
\text { external } \\
\text { forces } \\
W_{e x, a}^{(r)}(t)\end{array}$ & $\begin{array}{l}\text { Type of system behaviour } \\
\text { based on the energy exchange } \\
\text { with the environment }\end{array}$ \\
\hline 1 & $t \in(0,1]$ & $\begin{array}{l}E_{T, a}^{(1)}(t) \\
\text { given by } \\
\text { Eq. (30a) }\end{array}$ & $\begin{array}{l}P_{e x, a}^{(1)}(t) \text { given } \\
\text { by Eq. (31a) }\end{array}$ & $\begin{array}{l}W_{e x, a}^{(1)}(t) \\
\text { given by Eq. } \\
(32 \mathrm{a})\end{array}$ & $\begin{array}{l}\text { Perpetual machine of } 3^{\text {rd }} \text { kind, } \\
\text { and upon the source might be } \\
\text { a perpetual machine of } 2^{\text {nd }} \\
\text { kind }\end{array}$ \\
\hline
\end{tabular}




\begin{tabular}{|l|l|l|l|l|l|}
\hline $2 t \in(1,2]$ & $\begin{array}{l}E_{T, a}^{(1)}(t) \\
\text { given by } \\
\text { Eq. (30a) }\end{array}$ & $\begin{array}{l}P_{e x, a}^{(1)}(t) \text { given } \\
\text { by Eq. (31a) }\end{array}$ & $\begin{array}{l}W_{e x, a}^{(1)}(t) \\
\text { given by Eq. } \\
(32 \mathrm{a})\end{array}$ & $\begin{array}{l}\text { Perpetual machine of } 3^{\text {rd }} \text { kind, } \\
\text { and upon the source might be } \\
\text { a perpetual machine of } 2^{\text {nd }} \\
\text { kind }\end{array}$ \\
\hline 3 & $t \in(2,3]$ & $\begin{array}{l}E_{T, a}^{(2)}(t) \\
\text { given by } \\
\text { Eq. (30b) }\end{array}$ & $\begin{array}{l}P_{\text {ex,a }}^{(2)}(t) \text { given } \\
\text { by Eq. (31b) }\end{array}$ & $\begin{array}{l}W_{e x, a}^{(2)}(t) \\
\text { given by Eq. } \\
(32 \mathrm{~b})\end{array}$ & $\begin{array}{l}\text { Perpetual machine of } 3^{\text {rd }} \text { kind } \\
\text { for few time instants, with } \\
\text { potential to behave as } \\
\text { perpetual machine of } 2^{\text {nd }} \text { kind }\end{array}$ \\
\hline
\end{tabular}

Table 2. The type of system behavior based on the energy exchange with the environment, for each time interval, and referencing the explicit form of equations defining the kinetic energy, the external forces power, and work done.

\begin{tabular}{|c|c|c|c|c|}
\hline$M_{x}$ & $K_{x}$ & \multicolumn{1}{c|}{$C_{x}$} & \multicolumn{2}{|c|}{$F_{x, N L, i}$} \\
\hline$m_{1}=2000 \mathrm{~kg}$ & $k_{x, 1}=1 \cdot 10^{6} \mathrm{~N} / \mathrm{m}$ & $\begin{array}{l}c_{x, 1} \\
=1085.05 \mathrm{~N} \cdot \mathrm{s} / \mathrm{m}\end{array}$ & $k_{x, n l, 1}=1 \cdot 10^{5} \mathrm{~N}$ & $c_{x, n l, 1}=1085.05 \mathrm{~N} \cdot \mathrm{s}^{3} / \mathrm{m}^{3}$ \\
\hline$m_{2}=1000 \mathrm{~kg}$ & $k_{x, 2}=1.4 \cdot 10^{6} \mathrm{~N} / \mathrm{m}$ & $\begin{array}{l}c_{x, 2} \\
=1519.07 \mathrm{~N} \cdot \mathrm{s} / \mathrm{m}\end{array}$ & $k_{x, n l, 2}=-1.3 \cdot 10^{5} \mathrm{~N} / \mathrm{m}^{5}$ & $c_{x, n l, 2}=1519.07 \mathrm{~N} \cdot \mathrm{s}^{5} / \mathrm{m}^{5}$ \\
\hline$m_{3}=1500 \mathrm{~kg}$ & $k_{x, 3}=1.3 \cdot 10^{6} \mathrm{~N} / \mathrm{m}$ & $\begin{array}{l}c_{x, 3} \\
=1410.56 \mathrm{~N} \cdot \mathrm{s} / \mathrm{m}\end{array}$ & $k_{x, n l, 3}=1.2 \cdot 10^{5} \mathrm{~N}$ & $c_{x, n l, 3}=1410.56 \mathrm{~N}$ \\
\hline$m_{4}=1200 \mathrm{~kg}$ & $k_{x, 4}=1.2 \cdot 10^{6} \mathrm{~N} / \mathrm{m}$ & $\begin{array}{l}c_{x, 4} \\
=1302.06 \mathrm{~N} \cdot \mathrm{s} / \mathrm{m}\end{array}$ & $k_{x, n l, 4}=1 \cdot 10^{5} \mathrm{~N} / \mathrm{m}^{7}$ & $c_{x, n l, 4}=1302.06 \mathrm{~N}$ \\
\hline$m_{5}=1000 \mathrm{~kg}$ & - & - & - & - \\
\hline
\end{tabular}

Table 3. The mass, stiffness, damping matrix, and nonlinear forces values of the parameters.

Considering $1 \%$ damping ratio for the $2^{\text {nd }}$ natural frequency then the Rayleigh damping coefficient is $\beta_{1}=1.0850479 \cdot 10^{-3} \mathrm{~s}$, and is used in equation (30c) to obtain the values of the damping coefficients (Table 3 ). The nonlinear damping coefficients are of the same arithmetic values as the linear damping coefficients, and also, in smoothed dry friction nonlinear damping forces, the following value $b=10^{6} \mathrm{~s} / \mathrm{m}$ is used.

\begin{tabular}{|c|c|}
\hline$i$ & $\begin{array}{c}\omega_{i} \\
(\mathrm{rad} / \mathrm{s})\end{array}$ \\
\hline 1 & 0 \\
\hline 2 & 18.432 \\
\hline 3 & 34.164 \\
\hline 4 & 52.465 \\
\hline 5 & 61.023 \\
\hline
\end{tabular}


Table 4. The natural frequencies of the underlying linear system obtained by solving the linear eigenvalue problem.

\begin{tabular}{|c|c|c|c|c|c|}
\hline \multirow[b]{2}{*}{$\mathrm{i}$} & \multirow{2}{*}{$\begin{array}{c}\text { Time interval } \\
\text { (s) }\end{array}$} & \multicolumn{2}{|c|}{ ICs } & \multirow[b]{2}{*}{ External Forcing Parameters } & \multirow{2}{*}{$\begin{array}{l}w v_{x, i}(t) \\
(\mathrm{m} / \mathrm{s})\end{array}$} \\
\hline & & $\begin{array}{c}\dot{x}_{a, i}\left(t_{0}\right) \\
(\mathrm{m} / \mathrm{s})\end{array}$ & $\begin{array}{c}x_{a, i}\left(t_{0}\right) \\
(\mathrm{m})\end{array}$ & & \\
\hline \multirow{2}{*}{1} & \multirow{2}{*}{$t \in(0,1]$} & \multirow{2}{*}{0.000000} & \multirow{2}{*}{0.000000} & $\eta_{1}=5000 \mathrm{~N} / \mathrm{s}$ & \multirow{2}{*}{-} \\
\hline & & & & $c_{1}=10 \mathrm{~N}$ & \\
\hline \multirow{2}{*}{2} & \multirow{2}{*}{$t \in(1,2]$} & \multirow{2}{*}{1.255000} & \multirow{2}{*}{0.419167} & $\eta_{2}=-5000 \mathrm{~N} / \mathrm{s}$ & \multirow{2}{*}{-} \\
\hline & & & & $c_{2}=-10 \mathrm{~N}$ & \\
\hline \multirow{3}{*}{3} & \multirow{3}{*}{$t \in(2,3]$} & \multirow{3}{*}{-2.500000} & \multirow{3}{*}{0.005000} & $A_{e x}=137219.51526056 \mathrm{~N}$ & \multirow{3}{*}{0} \\
\hline & & & & $\omega_{e x}=\omega_{2}=18.43 \mathrm{rad} / \mathrm{s}$ & \\
\hline & & & & $\theta_{e x}=0 \mathrm{rad}$ & \\
\hline
\end{tabular}

Table 5. The initial conditions, the external forcing parameters, and the wave velocity of the train.

All the numerical simulations on this section with Scilab 5.5.2 64-bit [36] using 'Adams' solver, have been performed. The time step is $5 \cdot 10^{-4} \mathrm{~s}$, and the relative and absolute tolerance are $9 \cdot 10^{-15}$.

In Figure 2, the analytical displacements $\left(x_{\alpha}\right)$ and the numerical determined displacements of the two edges $\left(x_{1}, x_{5}\right)$ and the train's middle wagon $\left(x_{3}\right)$, are depicted. All of them look like they coincide, and this is certified by examining the values of the maximum absolute differences between the time series. More precisely, considering the maximum absolute differences between the time series of all the numerical determined displacements with the analytical solution, their maximum of these maximal values is $7.56 \times 10^{-12} \mathrm{~m}$. Therefore, the system's motion is described by the analytical solution, and it is in the exact augmented perpetual manifolds. The train's motion for the first $2 \mathrm{~s}$ that corresponds to the first two time intervals is clearly particle-rigid body curvilinear motions, and both are irreversible processes. The motion is a particle-standing wave in the third time interval, and it is a reversible process. The two magenta dash-dot lines indicate the time limits of two full periodic motions with a period of $0.34087784 \mathrm{~s}$. After the time instant designated by the $2^{\text {nd }}$ magenta dash-dot line, the motion still corresponds to a periodic motion but incomplete due to the integration time's finish. 


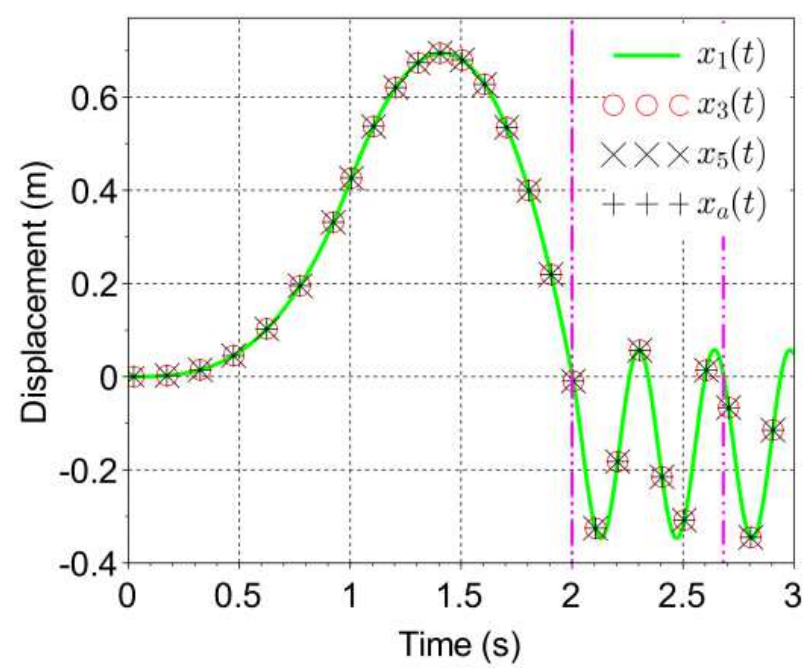

Figure 2. The displacements of 3 train wagons, these ones of the two wagons at the edges $\left(x_{1}, x_{5}\right)$ and of the middle wagon $\left(x_{3}\right)$, incorporating the analytical solution $\left(x_{\alpha}\right)$.

In Figure 3a, the numerical determined velocities of the wagons at the two edges $\left(\dot{x}_{1}, \dot{x}_{5}\right)$ and the middle wagon $\left(\dot{x}_{3}\right)$ of the train are depicted, incorporating the analytical velocity $\left(\dot{x}_{\alpha}\right)$. They seem that they coincide, and this is confirmed by examining the maximum absolute differences of the time series between the analytical velocity with the time-series of the velocities of all wagons of the train. Considering the maximum absolute differences between all the timeseries of the numerical determined velocities with the analytical velocity, the maximum of all of them is $1.81 \times 10^{-11} \mathrm{~m} / \mathrm{s}$, which certifies that the analytical solution describes the motion, and it is in exact augmented perpetual manifolds. The two magenta dash-dot lines are indicating the time limits of a periodic motion with two periods. The cyan regions in Figure 3a indicate the time intervals that the velocity is codirected with the external force by means that they have the same sign, and their product lead to positive values of power. The limits of these cyan regions can be obtained by identifying the zero crossings of the power that their exact determination is limited to the time resolution. They are determined by identifying adjacent points that the numerically determined power of the external forces is changing sign. Table 6 shows the numerically determined time intervals for positive power and the analytical determination of these time intervals. Based on the accuracy of the numerically determined time instants, that depends on the time resolution, which is $d t=5 \cdot 10^{-4} s$, they are in very good agreement with the analytical values. 
The external forces $\left(F_{x, 1}\right)$, for each time interval in Table 1 are indicated, with the general form given in Table A.1, and they are not state depended but only time depended. Therefore, for consistency using the time resolution of the numerical simulations, and the values of the parameters given in Table 5, they are easily plotted, and in Figure $3 \mathrm{~b}$ are depicted. The cyan areas indicate the time instants that the external force is co-directed with the velocities, and it is straightforward to be certified by a simple comparison of this plot with the plot of Figure 3a.

\begin{tabular}{|c|c|c|c|c|}
\hline $\mathrm{i}$ & $k$ & Analysis & $\begin{array}{l}\text { Analytical } \\
\text { Time interval with positive power } \\
\text { (s) }\end{array}$ & $\begin{array}{l}\text { Numerical } \\
\text { Time interval with positive power } \\
\text { with time resolution } 5 \cdot 10^{-4} s \\
\text { (s) }\end{array}$ \\
\hline 1 & - & (Ia) & {$[0,1]$} & {$[0,1]$} \\
\hline \multirow{2}{*}{2} & - & (If) & $(1.4150406,2]$ & \multirow{2}{*}{$(1.4155,2.0450)$} \\
\hline & 12 & (II) & $(2.00000000,2.04526705)$ & \\
\hline 3 & 13 & (II) & $(2.13048652,2.21570598)$ & $(2.1305,2.2155)$ \\
\hline 4 & 14 & (II) & $(2.30092544,2.38614490)$ & $(2.3010,2.3860)$ \\
\hline 5 & 15 & (II) & $(2.47136436,2.55658382)$ & $(2.4715,2.5565)$ \\
\hline 6 & 16 & (II) & $(2.64180328,2.72702274)$ & $(2.6420,2.7270)$ \\
\hline 7 & 17 & (II) & $(2.81224220,2.89746166)$ & $(2.8125,2.8970)$ \\
\hline 8 & 18 & (II) & $(2.98268112,3.00000000]$ & $(2.9830,3.0000]$ \\
\hline
\end{tabular}

Table 6. Analytical and numerically determined time intervals that the train is behaving as a perpetual machine of 3 rd kind.

In a-part of the theorem the individual terms of the internal forces are equal to zero. This is certified, using the numerically determined generalized coordinates and velocities, that all the individual internal forces given by the equations (40b-e) have been evaluated. The numerically determined generalized coordinates and velocities, used to determine all the individual internal forces given by the equations (40b-e). In Table 7 the maximum absolute values of the timeseries of each element of each internal force are shown. The maxima of the linear forces in the first two columns of Table 7 are shown. In the last two columns, the values of the maxima of the nonlinear forces are shown. The maximum of the maxima is in linear stiffness forces but in -9 order of magnitude, which is very minimal. In 
order to make sense of the determined values of the internal forces, the ratio of the time-series of them with the external forces given by the equations (40f) and also their explicit form is shown in Table 1, have been determined. The maximum absolute value of the timeseries ratios of each individual internal force with the external force in Table 8 is shown. The maximum of the maxima is of -10 order of magnitude; therefore all the internal forces are, due to numerical errors, almost zero. The eliminated internal forces certify the a-part of the theorem.

\begin{tabular}{|c|c|c|c|c|}
\hline$i$ & $\begin{array}{c}\max \left|F_{L, s t, i}\right| \\
(N)\end{array}$ & $\begin{array}{c}\max \left|F_{L, d, i}\right| \\
(N)\end{array}$ & $\begin{array}{c}\max \left|F_{N L, x, i}\right| \\
(N)\end{array}$ & $\begin{array}{c}\max \left|F_{N L, \dot{x}, i}\right| \\
(N)\end{array}$ \\
\hline 1 & $8.09 \cdot 10^{-9}$ & $1.84 \cdot 10^{-10}$ & $8.05 \cdot 10^{-10}$ & $5.26 \cdot 10^{-36}$ \\
\hline 2 & $7.16 \cdot 10^{-9}$ & $2.67 \cdot 10^{-10}$ & $8.05 \cdot 10^{-10}$ & $5.26 \cdot 10^{-36}$ \\
\hline 3 & $8.56 \cdot 10^{-9}$ & $3.13 \cdot 10^{-10}$ & $7.33 \cdot 10^{-10}$ & $1.17 \cdot 10^{-61}$ \\
\hline 4 & $8.03 \cdot 10^{-9}$ & $2.25 \cdot 10^{-10}$ & $7.33 \cdot 10^{-10}$ & $4.52 \cdot 10^{-10}$ \\
\hline 5 & $2.33 \cdot 10^{-10}$ & $1.51 \cdot 10^{-10}$ & $9.91 \cdot 10^{-107}$ & $4.52 \cdot 10^{-10}$ \\
\hline
\end{tabular}

Table 7. Maximum absolute value of the time series of each individual term of the internal forces for all the time instants.

\begin{tabular}{|c|c|c|c|c|}
\hline$i$ & $\max \left|\frac{F_{L, s t, i}}{F_{x, i}}\right|$ & $\max \left|\frac{F_{L, d, i}}{F_{x, i}}\right|$ & $\max \left|\frac{F_{N L, x, i}}{F_{x, i}}\right|$ & $\max \left|\frac{F_{N L, \dot{x}, i}}{F_{x, i}}\right|$ \\
\hline 1 & $4.35 \cdot 10^{-11}$ & $9.05 \cdot 10^{-13}$ & $4.34 \cdot 10^{-12}$ & $5.91 \cdot 10^{-39}$ \\
\hline 2 & $1.29 \cdot 10^{-10}$ & $4.74 \cdot 10^{-12}$ & $8.69 \cdot 10^{-12}$ & $1.18 \cdot 10^{-38}$ \\
\hline 3 & $9.64 \cdot 10^{-11}$ & $2.66 \cdot 10^{-12}$ & $5.40 \cdot 10^{-12}$ & $9.22 \cdot 10^{-64}$ \\
\hline 4 & $7.51 \cdot 10^{-11}$ & $9.14 \cdot 10^{-12}$ & $6.76 \cdot 10^{-12}$ & $1.84 \cdot 10^{-11}$ \\
\hline 5 & $2.02 \cdot 10^{-12}$ & $7.35 \cdot 10^{-12}$ & $3.37 \cdot 10^{-109}$ & $2.20 \cdot 10^{-11}$ \\
\hline
\end{tabular}

Table 8. Maximum absolute value of the ratio of the time series of each individual term of the internal forces for all the time instants with the timeseries of the external forces.

In Figure $3 \mathrm{c}$, the power of the external forces, of the analytical solution $\left(P_{e x, a}\right)$ and of the numerical simulation $\left(P_{e x}\right)$ are depicted, and it seems that they are in agreement. This is certified further on considering the maximum absolute difference of the their time-series, which has the minimal value of $7.994 \times 10^{-6} \mathrm{~W}$. The cyan areas indicate the time intervals with positive power, which can be easily certified that they correspond to the same time intervals of the other two figs $(3 a, b)$. 
In the next part of this section, the numerical values of the energies associated with each internal force are examined. The maximum absolute value of the linear's force potential energy is $1.21 \times 10^{-10} \mathrm{~J}$ and of the nonlinear force is $3.33 \times 10^{-81} \mathrm{~J}$, and their minimal values can be attributed to numerical errors. Therefore the part $b$ of the theorem, that the value of the potential energy, in the exact augmented perpetual manifolds remains constant, in this example, is verified.

The maximum value of the energy dissipated by the linear damping force is $1.022 \times 10^{-14} \mathrm{~J}$ and the nonlinear damping force is $5.567 \times 10^{-15} \mathrm{~J}$, and both of them are almost zero.

Therefore, in this example, the c-part of the theorem, in the exact augmented perpetual manifolds that there is no dissipation of energy, means that there is no energy loss in the form of heat. Therefore the process internally isentropic character is certified.

Also, the d-part of the theorem is implied, in case that the external forces that are applied by the environment are provided through an adiabatic process is straightforward to say that the perpetual mechanical system in an environment that is providing the external forces in an adiabatic manner are forming together an overall system that behaves as a perpetual machine of $2^{\text {nd }}$ kind. This mechanical system can behave as a perpetual machine of $2^{\text {nd }}$ kind in all-time instants, in the exact augmented perpetual manifolds with reversible and irreversible dynamics. Therefore the reversibility of the dynamics in the exact augmented perpetual manifolds is irrelevant.

In Figure $3 \mathrm{~d}$ the numerically determined kinetic energy $\left(E_{T}\right)$, mechanical energy $\left(E_{\text {mech }}\right)$, and the input energy $\left(E_{\text {in }}\right)$, are depicted, incorporating the analytically determined kinetic energy $\left(E_{T, a}\right)$. It seems that they are in good agreement. This can be certified further by examining the maximum absolute difference between the time-series of the analytically determined kinetic energy with the numerically determined kinetic energy with a minimal value of $4.422 \times 10^{-7} \mathrm{~J}$. Moreover, the maximum absolute difference between the numerically determined time-series of the kinetic energy with the input energy is minimal, with the value of $6.651 \times 10^{-7} \mathrm{~J}$. 

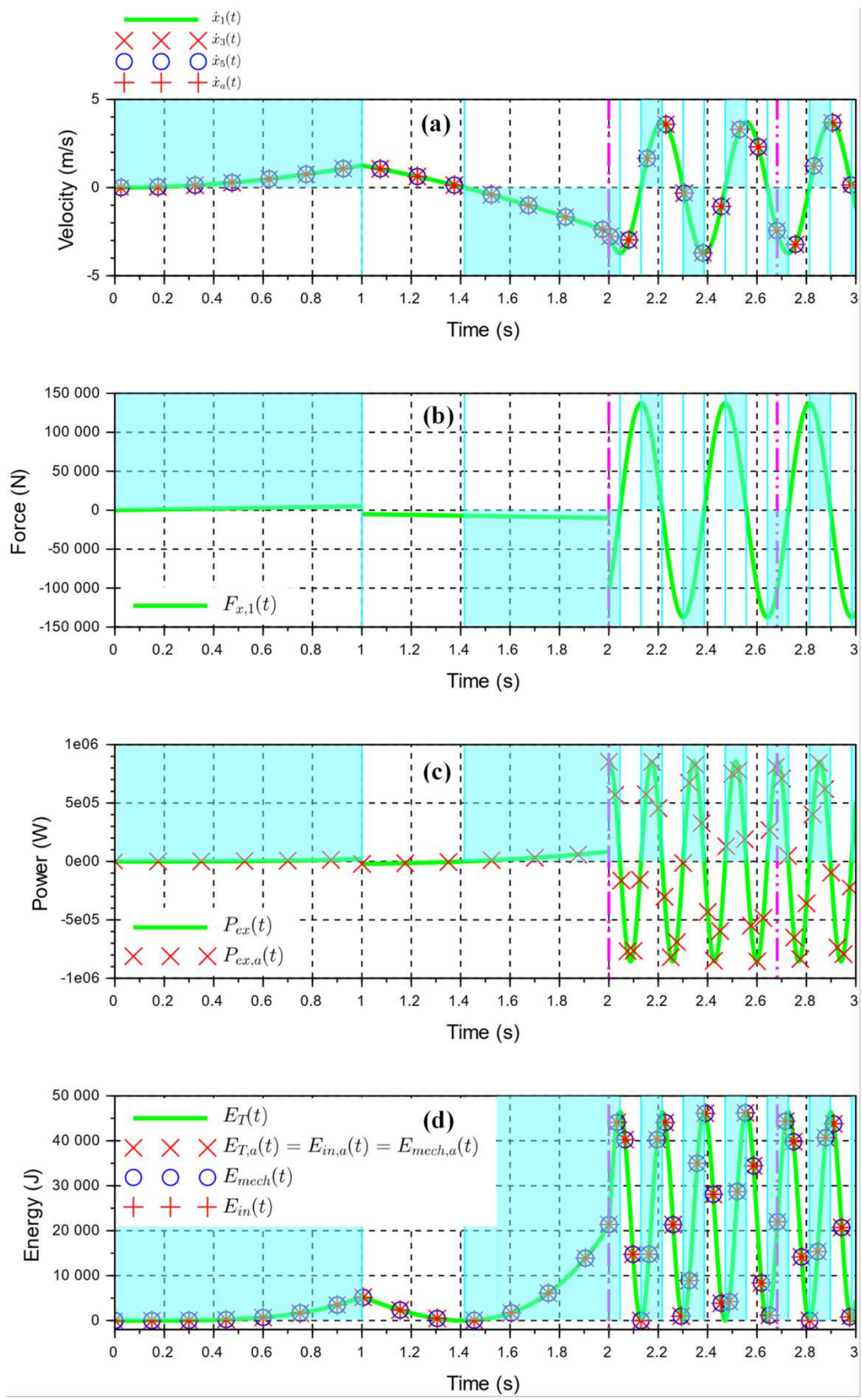

Figure 3. Analytical and numerical (index $-\alpha$ ) : (a) velocities, of 3 train wagons, these ones of the two wagons at the edges $\left(\dot{x}_{1}, \dot{x}_{5}\right)$ and of the middle wagon $\left(\dot{x}_{3}\right)$, (b) external force $\left(F_{x, 1}\right)$ that is applied in the first wagon, (c) external forces power $\left(P_{e x}\right)$, (d) energies of the mechanical system: kinetic energy $\left(E_{T}\right)$, input energy $\left(E_{\text {in }}\right)$, mechanical energy $\left(E_{\text {mech }}\right)$. The cyan regions indicate the 
time intervals that the mechanical system behaves as perpetual machine of $3^{\text {rd }}$ kind, by means the on these regions the kinetic energy is increasing, resulted by positive power of the external forces, due to co-directed (same sign, e.g. both positive or both negative) velocity with the external forces.

This certifies e-part of the theorem that the 'input' energy comprised of the initial kinetic energy with the work done by the external forces, in the augmented perpetual manifolds, is equal to the kinetic energy of the system.

The cyan regions correspond to time intervals with positive power, obtained by examining the time intervals that the velocity and the external forces are having the same sign. These cyan regions cover the same time intervals in all the other subfigures. In cyan regions easily can be concluded with the examination of Figure 3d, that the perpetual mechanical system is earning energy, since the kinetic energy and the mechanical energy on these time intervals are increasing. This is certifying the f-part of the theorem that co-directed force with velocity leads to the positive power of the external forces that the system's energy is increasing.

Examining the zone between the magenta dash-dot lines for the time instants $t \in[2,2.6818] s$, that the motion is periodic, it is clear that due to periodicity in some time intervals, the perpetual mechanical system is earning energy (cyan regions) and behaves as a perpetual machine of $3^{\text {rd }}$ kind. In some other time intervals, the system is losing energy, outside to the environment, therefore cannot behave as a perpetual machine of $3^{\text {rd }}$ kind, and this certifies the corollary.

\subsection{A two degrees of freedom non-perpetual mechanical system}

A 2-degrees of freedom non-perpetual mechanical system, as shown in Figure 4, including dry friction non-smooth external forces is considered. The equations of motion, setting the boundaries of the perpetual mechanical subsystem, and separating the forces associated with each element of the perpetual mechanical subsystem on the left side with other forces on the right-hand side, are given by,

$$
\begin{gathered}
{\left[\begin{array}{cc}
m_{r, 1} & 0 \\
0 & m_{r, 2}
\end{array}\right] \times\left\{\begin{array}{l}
\ddot{r}_{1} \\
\ddot{r}_{2}
\end{array}\right\}+\left[\begin{array}{cc}
k_{r} & -k_{r} \\
-k_{r} & k_{r}
\end{array}\right] \times\left\{\begin{array}{l}
r_{1} \\
r_{2}
\end{array}\right\}+\left[\begin{array}{cc}
c_{r} & -c_{r} \\
-c_{r} & c_{r}
\end{array}\right] \times\left\{\begin{array}{l}
\dot{r}_{1} \\
\dot{r}_{2}
\end{array}\right\}+} \\
+\left\{\begin{array}{l}
k_{n l, r} \cdot\left(r_{1}-r_{2}\right)^{3}+c_{n l, r} \cdot \tanh \left(b \cdot\left(\dot{r}_{1}-\dot{r}_{2}\right)\right)+g_{1} \cdot\left(r_{1}-r_{2}\right)^{2} \cdot\left(\dot{r}_{1}-\dot{r}_{2}\right) \\
k_{n l, r} \cdot\left(r_{2}-r_{1}\right)^{3}+c_{n l, r} \cdot \tanh \left(b \cdot\left(\dot{r}_{2}-\dot{r}_{1}\right)\right)+g_{1} \cdot\left(r_{2}-r_{1}\right)^{2} \cdot\left(\dot{r}_{2}-\dot{r}_{1}\right)
\end{array}\right\}= \\
=\left[\begin{array}{cc}
m_{r, 1} & 0 \\
0 & m_{r, 2}
\end{array}\right] \times\left\{\begin{array}{l}
\ddot{r}_{1} \\
\ddot{r}_{2}
\end{array}\right\}+\left\{F_{2 d, L, s t}\right\}+\left\{F_{2 d, L, d}\right\}+\left\{F_{2 d, N L, r}\right\}+\left\{F_{2 d, N L, \dot{r}}\right\}+
\end{gathered}
$$




$$
+\left\{F_{2 d, N L, g}\right\}=\left\{\begin{array}{l}
f_{1}\left(t, r_{1}, r_{2}, \dot{r}_{1}, \dot{r}_{2}\right)+f_{d, 1}\left(\dot{r}_{1}\right) \\
f_{2}\left(t, r_{1}, r_{2}, \dot{r}_{1}, \dot{r}_{2}\right)+f_{d, 2}\left(\dot{r}_{2}\right)
\end{array}\right\}=\left\{\begin{array}{l}
F_{\text {ext, } 1}\left(t, r_{1}, r_{2}, \dot{r}_{1}, \dot{r}_{2}\right) \\
F_{\text {ext }, 2}\left(t, r_{1}, r_{2}, \dot{r}_{1}, \dot{r}_{2}\right)
\end{array}\right\},
$$

whereas in the last line the external forces are defined and in the previous line the different types of the internal forces in condensed vector form are shown. The external forces are given by,

$$
\begin{gathered}
f_{1}\left(t, r_{1}, r_{2}, \dot{r}_{1}, \dot{r}_{2}\right)=-\alpha \cdot \dot{r}_{1}-\mu \cdot r_{1}-\mu_{n l} \cdot r_{1}^{3}+f_{r}(t), \\
f_{2}\left(t, r_{1}, r_{2}, \dot{r}_{1}, \dot{r}_{2}\right)=-\frac{m_{r, 2}}{m_{r, 1}} \cdot \alpha \cdot \dot{r}_{2}-\frac{m_{r, 2}}{m_{r, 1}} \cdot \mu \cdot r_{2}-\frac{m_{r, 2}}{m_{r, 1}} \cdot \mu_{n l} \cdot r_{2}^{3}+\frac{m_{r, 2}}{m_{r, 1}} \cdot f_{r}(t),(
\end{gathered}
$$

and,

$$
\begin{aligned}
& f_{d, 1}\left(\dot{r}_{1}\right)=-\frac{\mu_{1}^{s} \cdot F_{1}^{N}}{1+\delta \cdot\left|\dot{r}_{1}\right|} \cdot \operatorname{sign}\left(\dot{r}_{1}\right), \\
& f_{d, 2}\left(\dot{r}_{2}\right)=-\frac{\mu_{1}^{s} \cdot F_{2}^{N}}{1+\delta \cdot\left|\dot{r}_{2}\right|} \cdot \operatorname{sign}\left(\dot{r}_{2}\right) .
\end{aligned}
$$

The application of the theorem proved in [21] on this mechanical system, by neglecting the external forces on the right-hand side of equations (45a), requires that the system of differential equations with the remaining terms, that are given by,

$$
\begin{gathered}
{\left[\begin{array}{cc}
m_{r, 1} & 0 \\
0 & m_{r, 2}
\end{array}\right] \times\left\{\begin{array}{l}
\ddot{r}_{1} \\
\ddot{r}_{2}
\end{array}\right\}+\left[\begin{array}{cc}
k_{r} & -k_{r} \\
-k_{r} & k_{r}
\end{array}\right] \times\left\{\begin{array}{l}
r_{1} \\
r_{2}
\end{array}\right\}+\left[\begin{array}{cc}
c_{r} & -c_{r} \\
-c_{r} & c_{r}
\end{array}\right] \times\left\{\begin{array}{l}
\dot{r}_{1} \\
\dot{r}_{2}
\end{array}\right\}+} \\
+\left\{\begin{array}{c}
k_{n l, r} \cdot\left(r_{1}-r_{2}\right)^{3}+c_{n l, r} \cdot \tanh \left(b \cdot\left(\dot{r}_{1}-\dot{r}_{2}\right)\right)+g_{1} \cdot\left(r_{1}-r_{2}\right)^{2} \cdot\left(\dot{r}_{1}-\dot{r}_{2}\right) \\
k_{n l, r} \cdot\left(r_{2}-r_{1}\right)^{3}+c_{n l, r} \cdot \tanh \left(b \cdot\left(\dot{r}_{2}-\dot{r}_{1}\right)\right)+g_{1} \cdot\left(r_{2}-r_{1}\right)^{2} \cdot\left(\dot{r}_{2}-\dot{r}_{1}\right)
\end{array}\right\}= \\
=\{0\},
\end{gathered}
$$

should form a perpetual mechanical system. A perpetual mechanical system is defined, when accepts as perpetual points, the generalized coordinates and velocities for rigid body motion. Therefore the following correlation of generalized coordinates and velocities,

$$
r_{1}=r_{2}
$$

and

$$
\dot{r}_{1}=\dot{r}_{2} \neq 0,
$$

perpetual mechanical system, leads to zero accelerations and jerks of this system. Considering equations (46b-c) in the equation (46a) leads to,

$$
\left[\begin{array}{cc}
m_{r, 1} & 0 \\
0 & m_{r, 2}
\end{array}\right] \times\left\{\begin{array}{l}
\ddot{r}_{1} \\
\ddot{r}_{2}
\end{array}\right\}=\{0\} \Rightarrow \ddot{r}_{1}=\ddot{r}_{2}=0 .
$$

The equations of jerks arise by taking the time derivative of equations (46a) and they are given by,

$$
\left[\begin{array}{cc}
m_{r, 1} & 0 \\
0 & m_{r, 2}
\end{array}\right] \times\left\{\begin{array}{l}
\dddot{r}_{1} \\
\dddot{r}_{2}
\end{array}\right\}+\left[\begin{array}{cc}
k_{r} & -k_{r} \\
-k_{r} & k_{r}
\end{array}\right] \times\left\{\begin{array}{l}
\dot{r}_{1} \\
\dot{r}_{2}
\end{array}\right\}+\left[\begin{array}{cc}
c_{r} & -c_{r} \\
-c_{r} & c_{r}
\end{array}\right] \times\left\{\begin{array}{l}
\ddot{r}_{1} \\
\ddot{r}_{2}
\end{array}\right\}+
$$




$$
\begin{gathered}
+\left\{\begin{array}{c}
3 \cdot k_{n l, r} \cdot\left(r_{1}-r_{2}\right)^{2} \cdot\left(\dot{r}_{1}-\dot{r}_{2}\right)+b \cdot c_{n l, r} \cdot \operatorname{sech}^{2}\left(b \cdot\left(\dot{r}_{1}-\dot{r}_{2}\right)\right) \cdot\left(\ddot{r}_{1}-\ddot{r}_{2}\right) \\
3 \cdot k_{n l, r} \cdot\left(r_{2}-r_{1}\right)^{2} \cdot\left(\dot{r}_{2}-\dot{r}_{1}\right)+b \cdot c_{n l, r} \cdot \operatorname{sech}^{2}\left(b \cdot\left(\dot{r}_{2}-\dot{r}_{1}\right)\right) \cdot\left(\ddot{r}_{2}-\ddot{r}_{1}\right)
\end{array}\right\}+ \\
+\left\{\begin{array}{l}
2 \cdot g_{1} \cdot\left(r_{1}-r_{2}\right) \cdot\left(\dot{r}_{1}-\dot{r}_{2}\right)^{2}+g_{1} \cdot\left(r_{1}-r_{2}\right)^{2} \cdot\left(\ddot{r}_{1}-\ddot{r}_{2}\right) \\
2 \cdot g_{1} \cdot\left(r_{2}-r_{1}\right) \cdot\left(\dot{r}_{2}-\dot{r}_{1}\right)^{2}+g_{1} \cdot\left(r_{2}-r_{1}\right)^{2} \cdot\left(\ddot{r}_{2}-\ddot{r}_{1}\right)
\end{array}\right\}= \\
=\{0\} .
\end{gathered}
$$

Considering the equations (46b-c) in the equations of jerks (46e) lead to,

$$
\left[\begin{array}{cc}
m_{r, 1} & 0 \\
0 & m_{r, 2}
\end{array}\right] \times\left\{\begin{array}{c}
\dddot{r}_{1} \\
\dddot{r}_{2}
\end{array}\right\}=\{0\} \Rightarrow \dddot{r}_{1}=\dddot{r}_{2}=0
$$

Since the replacement of displacements and velocities given by equations $(46 \mathrm{~b}, \mathrm{c})$ to the equations of accelerations and jerks lead, the system with motion described by the equation (46a) is a perpetual mechanical system. Also, it is straight forward to show that in the exact augmented perpetual manifolds all the individual linear internal forces of equation (46a) are zero, and using also equation (9) all the vectors of the nonlinear forces they are zero, therefore they are orthogonal to each other, and the criteria of the theorem of section 2 are fulfilled.

\section{Boundary limits of the Perpetual Mechanical Subsystem}

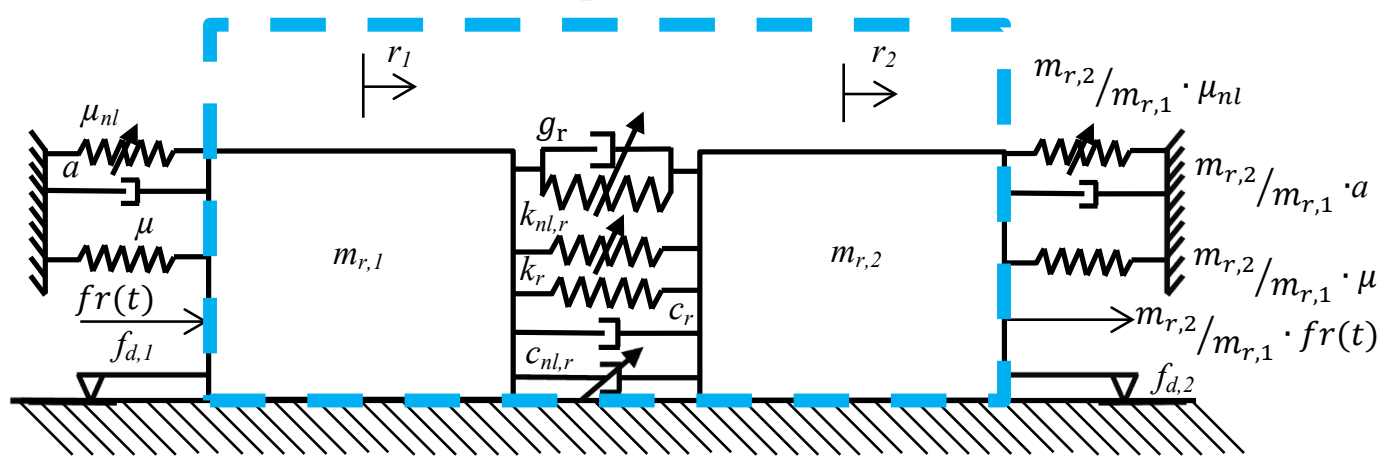

Figure 4. The configuration of the 2-dof non-perpetual mechanical system, indicating with cyan dashed line the boundaries of the perpetual mechanical subsystem.

In the right-hand side of equations (45a), there are nonsmooth forces with terms defined by equations (45d-e) that correspond to dry friction. Each dry friction force has a discontinuity when the associated velocity is zero [32].

Considering the first as $k$-mass, then from equations $(45 \mathrm{~b}, \mathrm{c})$ arise that,

$$
f_{2}\left(t, r_{a}, r_{a}, \dot{r}_{a}, \dot{r}_{a}\right)=\frac{m_{r, 2}}{m_{r, 1}} \cdot f_{1}\left(t, r_{a}, r_{a}, \dot{r}_{a}, \dot{r}_{a}\right)
$$

The normal forces amplitude in dry friction components, are correlated by,

$$
F_{2}^{N}=m_{r, 2} \cdot g=\frac{m_{r, 2}}{m_{r, 1}} \cdot F_{1}^{N} .
$$


Exact rigid body motion solution considering equation (48b) in the equations $(45 \mathrm{~d}, \mathrm{e})$ leads that,

$$
f_{d, 2}\left(\dot{r}_{a}\right)=\frac{m_{r, 2}}{m_{r, 1}} \cdot\left(-\frac{\mu_{1}^{s} \cdot F_{1}^{N}}{1+\delta \cdot\left|\dot{r}_{a}\right|} \cdot \operatorname{sign}\left(\dot{r}_{a}\right)\right)=\frac{m_{r, 2}}{m_{r, 1}} \cdot f_{d, 1}\left(\dot{r}_{a}\right)
$$

Therefore the external forces, for an exact rigid body motion solution, considering equations $(48 \mathrm{a}, \mathrm{c})$ in equation $(45 \mathrm{a})$, are correlated by,

$$
F_{\text {ext }, 2}\left(t, r_{a}, r_{a}, \dot{r}_{a}, \dot{r}_{a}\right)=\frac{m_{r, 2}}{m_{r, 1}} \cdot F_{e x t, 1}\left(t, r_{a}, r_{a}, \dot{r}_{a}, \dot{r}_{a}\right),
$$

which is exactly in the form of equation (5) that leads to an exact augmented perpetual manifolds solution.

The solution of the system (45a-e), in the augmented perpetual manifolds is given by the following form of the equation (6),

$$
\begin{gathered}
\ddot{r}_{a}(t)=\frac{1}{m_{r, 1}}\left(-\alpha \cdot \dot{r}_{a}-\mu \cdot r_{a}-\mu_{n l} \cdot r_{a}^{3}+f_{r}(t)-\frac{\mu_{1}^{s, r} \cdot F_{1}^{N, r}}{1+\delta \cdot\left|\dot{r}_{a}\right|} \cdot \operatorname{sign}\left(\dot{r}_{a}\right)\right)= \\
=\frac{1}{m_{r, 1}} \cdot F_{\text {ext }, a} .
\end{gathered}
$$

In this example the considered time depended external force is given by,

$$
f_{r}(t)=A_{e x} \cdot \sin \left(\omega_{e x} \cdot t\right) .
$$

This characteristic differential equation (49) is forming ROM of the original system, and, herein, numerically is solved.

The considered two masses have values $m_{r, 1}=2000 \mathrm{~kg}$ and $m_{r, 2}=1000 \mathrm{~kg}$, and for the perpetual mechanical system the linear stiffness is $k_{r}=10^{6} \mathrm{~N} / \mathrm{m}$, the nonlinear stiffness is $k_{n l, r}=5 \cdot 10^{5} \mathrm{~N} / \mathrm{m}^{3}$ and, the parameter that defines the nonlinear generalized force is $g_{1}=10^{6} \mathrm{~N} \cdot \mathrm{s} / \mathrm{m}^{3}$. The considered damping coefficient is $c_{r}=516.398 \mathrm{~N} \cdot \mathrm{s} / \mathrm{m}$, and for the nonlinear damping coefficient the same numerical value is considered with $c_{n l, r}=516.398 \mathrm{~N}$, and also $b=10^{6} \mathrm{~s} / \mathrm{m}$ (significantly high to approximate well a dry friction force). The parameters defining the other elements considered in external forcing are a linear spring with stiffness $\mu=3.2 \cdot 10^{6} \mathrm{~N} / \mathrm{m}$, a nonlinear spring with stiffness $\mu_{n l}=3.2 \cdot 10^{6} \mathrm{~N} / \mathrm{m}^{3}$, a viscous linear damper with damping coefficient $\alpha=$ $3000 \mathrm{~N} \cdot \mathrm{s} / \mathrm{m}$ and static friction coefficient $\mu_{1}^{s, r}=0.1$ and dynamic friction parameter $\delta=3 \mathrm{~s} / \mathrm{m}$. The initial conditions are $\left(r_{1}(0), r_{2}(0), \dot{r}_{1}(0), \dot{r}_{2}(0)\right)=$ $(1 \mathrm{~m}, 1 \mathrm{~m}, 0 \mathrm{~m} / \mathrm{s}, 0 \mathrm{~m} / \mathrm{s})$, and the time depended external force is having amplitude $A_{\text {ex }}=1 \cdot 10^{6} \mathrm{~N}$, and excitation frequency $\omega_{\text {ex }}=38.73 \mathrm{rad} / \mathrm{s}$. 
The numerical simulations for this section were performed using Scilab 5.5.2 64bit [36] with the 'Adams' solver. The time step is $5 \cdot 10^{-4} \mathrm{~s}$, and the relative and the absolute tolerance are $5 \cdot 10^{-14}$.

In Figure 5a, the displacements of the two masses incorporating the ROM solution are depicted. They look like that they are in good agreement, and this can be verified by examining the maximum of the absolute difference between each one of the two masses displacements with the ROM solution that the maximum of their maxima is having the minimal value of $1.932 \times 10^{-11} \mathrm{~m}$. Therefore the system's solution is in an exact augmented perpetual manifold. Moreover, this can be certified further by examining Figure 5b, whereas the two masse's velocities and the ROM solution are depicted. It seems that they are in good agreement. This can be certified by examining the maximum absolute difference between all the time-series of two masse's velocities with the ROM velocity, that the maximum of their maxima is having the minimal value of $1.348 \times 10^{-9} \mathrm{~m} / \mathrm{s}$. The zero values of the velocities at certain time instants in Figure $5 \mathrm{~b}$ means that the solution is crossing the discontinuities of the vector field.

Moreover, using the numerically determined generalized coordinates and velocities, the internal forces of the perpetual mechanical system defined by the system of equation (45a) are evaluated. According to the theoretical part, each individual term of internal forces within the boundaries of the perpetual mechanical system is equal to zero. In Table 9, the maximum absolute values of the timeseries of each individual term of the internal forces are determined. In the first two columns, the values of the maxima of the linear forces are indicated. In the last three columns, there are the values of the maxima of the nonlinear forces. These values of internal forces are very minimal, with a maximum of maxima of 6 order of magnitude. Judgment of the significance of the maximum values can be done by considering the ratio of the timeseries of each individual force with the timeseries of the total value of the external forces (given by eq. $45 \mathrm{a}$ ), as in Table 10 is shown. The maximum of the maxima of the absolute values of each individual ratio has the minimal -10 order of magnitude, corresponding to the nonlinear damping forces. The nonzero values can be attributed to numerical errors, and through these minimal values, the a-part of the theorem is certified. In Figure 6a, the power of the external forces obtained by the original system and the ROM solution is depicted, and it seems that they are in good agreement. This 
can be certified by examining the maximum absolute difference between the original system external forces power with this one from the ROM solution with a minimal value of $1.234 \times 10^{-2} \mathrm{~W}$. The cyan regions indicate the time intervals that the power of the external forces is positive, and they are obtained by examining adjacent points of the original system time-series of the power that they have opposite sign.

The maximum absolute value of the potential energy associated with the linear forces is $1.541 \times 10^{-25} \mathrm{~J}$, and with nonlinear forces is $1.187 \times 10^{-56}$. Therefore they are almost zero.

The almost zero values of the elastic potential energies lead to the conclusion that the b-part of the theorem on this overall non-perpetual mechanical system is verified.

The maximum absolute value of the dissipated energy a) through the linear dissipative forces is $3.673 \times 10^{-18} \mathrm{~J}$, and $\mathrm{b}$ ) through the nonlinear dissipative forces is $3.073 \times 10^{-12} \mathrm{~J}$. The maximum absolute value of the energy associated with the generalized force is $1.924 \times 10^{-44} \mathrm{~J}$, practically zero. The non-zero but minimal values of each dissipative internal force's energy can be attributed to numerical errors, and c-part of the theorem that in augmented perpetual manifolds, the process is isentropic is verified. Although the overall mechanical system is not perpetual, limiting the boundaries to the perpetual mechanical subsystem, in the case of exact augmented perpetual manifolds solutions, the perpetual mechanical subsystem has zero change in the internal entropy. Due to the dissipative forces outside of its boundary, the under consideration mechanical system cannot behave as a perpetual machine of $2^{\text {nd }}$ kind. Nevertheless, within its boundaries can behave as a perpetual machine of $3^{\text {rd }}$ kind and this can be certified by examining Figure $6 \mathrm{~b}$. Figure (6b) depicts the kinetic energy $\left(E_{2 d, T}\right)$, the mechanical energy $\left(E_{2 d, m e c h}\right)$, and the input energy $\left(E_{2 d, i n}\right)$, obtained from the numerically integrated original system, and the kinetic energy $\left(E_{2 d, T, R O M}\right)$, and the input energy $\left(E_{2 d, i n, R O M}\right)$ obtained from the ROM solution. It seems that they are all the same, and this can be certified further by examining their differences. The maximum absolute difference between the original (ROM) system's kinetic energy with the input energy is $1.845 \times 10^{-4} \mathrm{~J}\left(9.469 \times 10^{-6} \mathrm{~J}\right)$, and these minimal values certify the e-part of the theorem. The maximum absolute difference between the kinetic energy obtained by the original system with the kinetic energy 
obtained by ROM is $1.142 \times 10^{-4} \mathrm{~J}$. Considering the energies in Figure $6 \mathrm{~b}$ are in the $6^{\text {th }}$ order of magnitude; therefore, these differences are minimal and can be attributed to numerical errors.
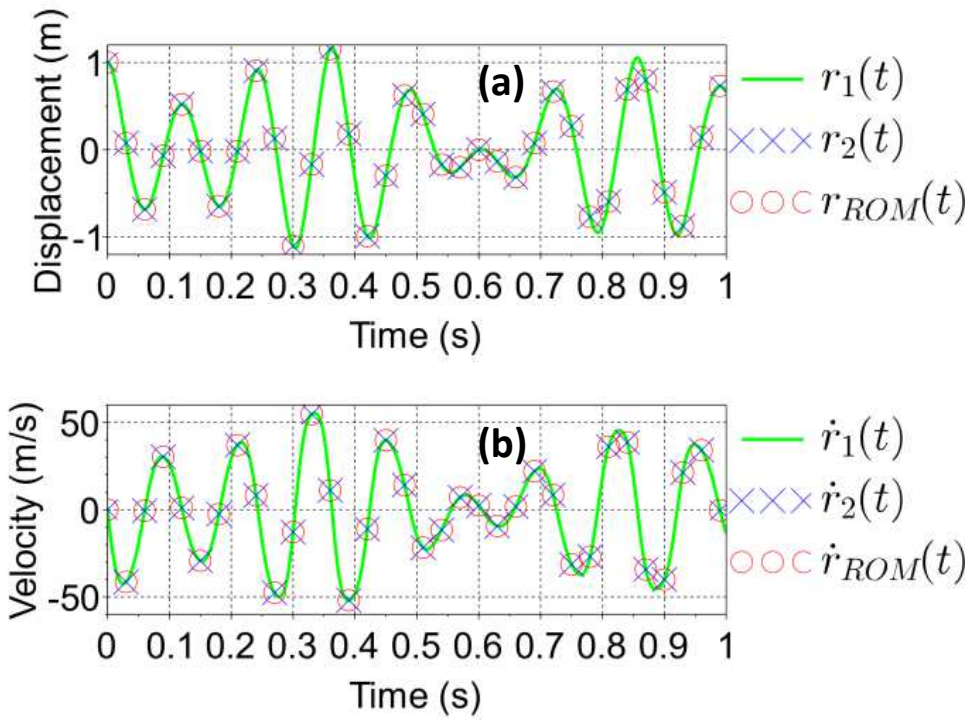

Figure 5. The numerically determined, (a) displacements of the original system with the ROM solution, (b) velocities of the original system with the ROM solution, verifying that the system's solution is in the exact augmented perpetual manifolds.

\begin{tabular}{|c|c|c|c|c|c|}
\hline$i$ & $\begin{array}{c}\max \left|F_{2 d, L, s t, i}(t)\right| \\
(N)\end{array}$ & $\begin{array}{c}\max \left|F_{2 d, L, d, i}(t)\right| \\
(N)\end{array}$ & $\begin{array}{c}\max \left|F_{2 d, N L, r, i}(t)\right| \\
(N)\end{array}$ & $\begin{array}{c}\max \left|F_{2 d, N L, \dot{r}, i}(t)\right| \\
(N)\end{array}$ & $\begin{array}{c}\max \left|F_{2 d, N L, g, i}(t)\right| \\
(N)\end{array}$ \\
\hline 1 & $5.55 \cdot 10^{-10}$ & $7.34 \cdot 10^{-12}$ & $8.55 \cdot 10^{-41}$ & $7.34 \cdot 10^{-6}$ & $3.55 \cdot 10^{-39}$ \\
\hline 2 & $5.55 \cdot 10^{-10}$ & $7.34 \cdot 10^{-12}$ & $8.55 \cdot 10^{-41}$ & $7.34 \cdot 10^{-6}$ & $3.55 \cdot 10^{-39}$ \\
\hline
\end{tabular}

Table 9. Maximum absolute value of the time series of each individual term of the internal forces for all the time instants.

\begin{tabular}{|c|c|c|c|c|c|}
\hline$i$ & $\max \left|\frac{F_{2 d, L, s t, i}(t)}{F_{\text {ext }, i}(t)}\right|$ & $\max \left|\frac{F_{2 d, L, d, i}(t)}{F_{\text {ext }, i}(t)}\right|$ & $\max \left|\frac{F_{N L, r, i}(t)}{F_{\text {ext }, i}(t)}\right|$ & $\max \left|\frac{F_{N L, \dot{r}, i}(t)}{F_{\text {ext }, i}(t)}\right|$ & $\max \left|\frac{F_{N L, \mathrm{~g}, i}(t)}{F_{\text {ext }, i}(t)}\right|$ \\
\hline 1 & $1.25 \cdot 10^{-13}$ & $2.86 \cdot 10^{-16}$ & $6.94 \cdot 10^{-45}$ & $2.86 \cdot 10^{-10}$ & $8.38 \cdot 10^{-44}$ \\
\hline 2 & $2.50 \cdot 10^{-13}$ & $5.73 \cdot 10^{-16}$ & $1.39 \cdot 10^{-44}$ & $5.73 \cdot 10^{-10}$ & $1.68 \cdot 10^{-43}$ \\
\hline
\end{tabular}

Table 10. Maximum absolute value of the ratio of the time series of each individual term of the internal forces for all the time instants with the timeseries of the external forces. 


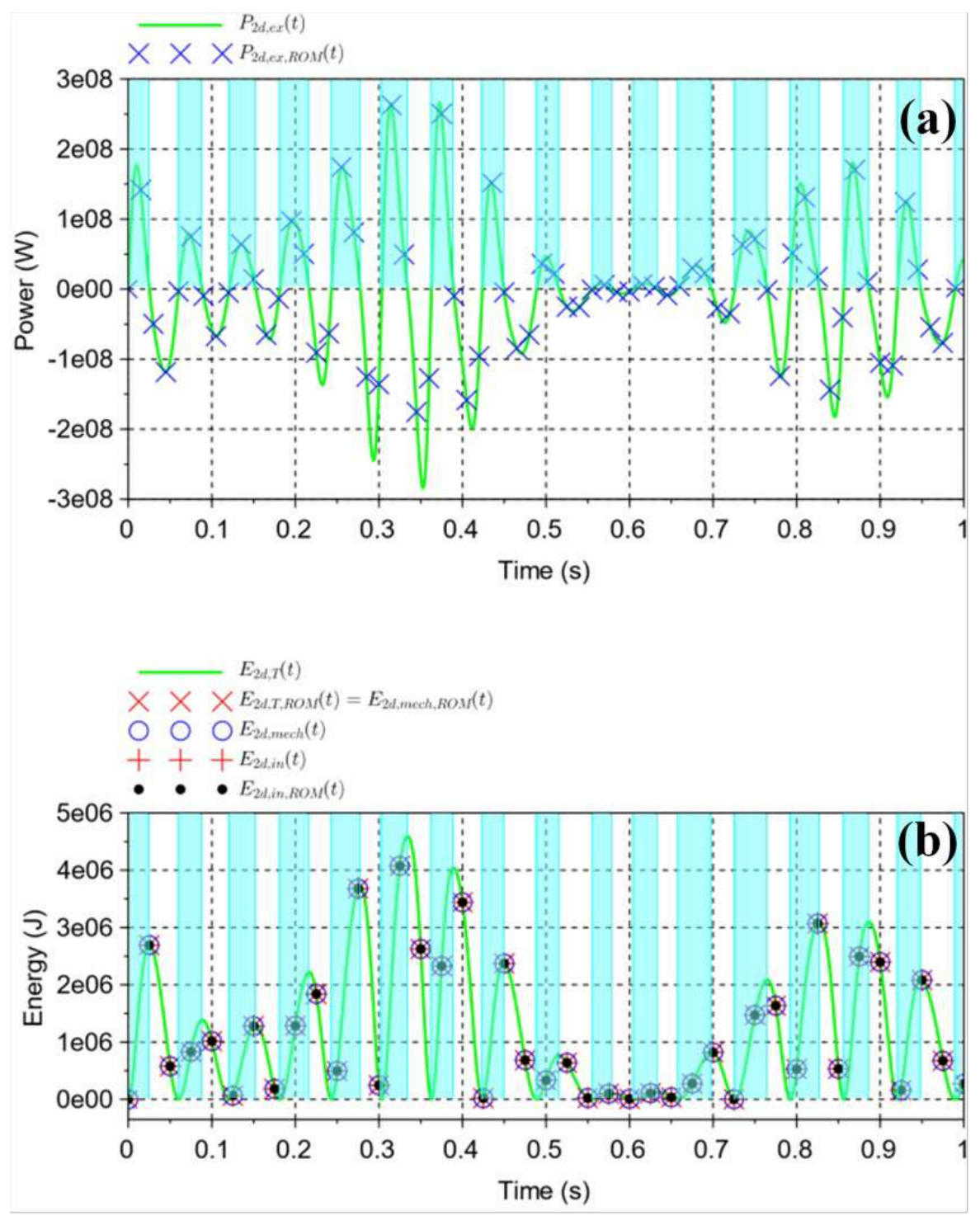

Figure 6. (a) The external forcing power of the original system and of the ROM solution. (b) The energies of the original system and of the ROM solution: kinetic energy, input energy, mechanical energy. The cyan regions indicate the time intervals that the mechanical energy of the system is increasing due to positive power of the external forces.

Moreover, on the time intervals indicated with cyan regions, the work done by the sum of the external forces becomes kinetic energy to the perpetual mechanical subsystem. Therefore, the perpetual mechanical subsystem behaves as a perpetual machine of $3^{\text {rd }}$ kind on these time intervals, and this certifies f-part of the theorem. Although the overall mechanical system is not perpetual, limiting the boundaries to the perpetual mechanical subsystem, the theorem and the corollary are certified. 


\section{CONCLUSIONS}

As a continuation of previous work relative to perpetual mechanical systems and their motion in the exact augmented perpetual manifolds, a theorem is proved, mainly stating that in motions described by exact augmented perpetual manifolds, each term of the individual the internal forces is zero, the internal entropy of the perpetual mechanical system and the elastic potential function remain constant. Further on, this conclusion leads to the conditions that a perpetual mechanical system can behave as a perpetual machine of $2^{\text {nd }}$ kind and $3^{\text {rd }}$ kind. Therefore the violation of the $2^{\text {nd }}$ law of thermodynamics for mechanical systems with solutions in the exact augmented perpetual manifolds is shown. Theorem's outcome is that there is no need for periodic processes for a mechanical system to behave as a perpetual machine of $2^{\text {nd }}$ kind. Therefore, the well-known requirement of the reversibility of the processes for the optimal behavior of mechanical systems with the view of the $2^{\text {nd }}$ law of thermodynamics as it is shown, it is not valid, and "the arrow of time' is not necessarily related with the entropy of a mechanical system. A corollary is proved that in periodic exact augmented perpetual manifolds solutions, the perpetual mechanical system cannot behave as a perpetual machine of $3^{\text {rd }}$ kind for all the time instants.

The theorem is verified with the analytical determination of the kinetic energy, the external forces power, and the external forces work done in the exact augmented perpetual manifolds for two types of external forces. A preliminary theoretical investigation for the conditions, through some analysis of these two types of external force's power, that a perpetual mechanical system behaves as a perpetual machine of $3^{\text {rd }}$ kind is performed.

In the last section, the validity of all the theoretical findings in two mechanical systems is examined, in a perpetual mechanical system and in a non-perpetual mechanical system that includes a perpetual mechanical subsystem. The theorem, the corollary, and the mathematical formalism for the two types of forces through these examples numerically verified with a very good agreement between the theoretical and numerical results.

This work is significant in physics and mechanical engineering. In physics relevant to the $2^{\text {nd }}$ law of thermodynamics, which requires reversibility of dynamics for the isentropic process and also the perpetual machine's existence was not so far under consideration. 
In mechanical engineering, machines operating without any internal forces are the ultimate ones since there is no degradation by internal stresses, and of course, the perpetual machines are the machines with the ultimate operation with zero loss of energy. Therefore the outcome is also of very high importance in mechanical engineering.

A continuation of this work can be done in several directions, e.g., statistical mechanics view of the dynamics in augmented perpetual manifolds, design of machines with zero internal forces, design of perpetual machines of $2^{\text {nd }}$ kind, the examination of the conditions that different type of external forces provides positive power to the system, and many others different directions.

\section{Declaration}

\section{Funding}

The author did not receive support from any organization for the submitted work.

\section{Conflicts of interest/Competing interests}

Just a start of this work with some examples of numerical simulations was done during Christmas holidays of 2019 while the author was an employee of the School of Engineering, College of Science at the University of Lincoln (UK). More precisely in a few simulations and draft plots of a simple nonlinear system, that is not included herein, the equality between the external forces work done with the kinetic energy of the system, is examined.

\section{Availability of data and material}

Data and material are available upon request to the author.

\section{Code availability}

All written codes are available upon request to the author.

\section{Ethics approval}

Nothing to declare for approval. 


\section{References}

1. Prasad A. Existence of perpetual points in nonlinear dynamical systems and its applications, International Journal of Bifurcation and Chaos Vol. 25, 1530005, (2015). DOI: 10.1142/S0218127415300050.

2. Brzeski, P, Virgin, L N. Experimental Investigation of perpetual points in mechanical systems, Nonlinear Dynamics; 90: 2917-2928 (2017).

3. Dudkowski, D, Prasad, A, Kapitaniak, T. Perpetual points and periodic perpetual loci in maps, Chaos, 26, 103103 (2016). https://doi.org/10.1063/1.4964266

4. Prasad, A. A note on topological conjugacy for perpetual points, International Journal of Nonlinear Science, 21: 60-64 (2016).

5. Dudkowski, D, Prasad, A, Kapitaniak, T. Perpetual points and hidden attractors in dynamical systems, Physics Letters A; 379: 2591-2596, (2015).

6. Dudkowski, D, Jafari, S, Kapitaniak, T, Kuznetsov, N V, Leonov, GA, Prasad, A. Hidden attractors in dynamical systems, Physics Reports, Vol. 637, pp. 1$50,(2016)$.

7. Dudkowski, D., Prasad, A., Kapitaniak, T. Perpetual Points: New Tool for Localization of Coexisting Attractors in Dynamical Systems," Int. J. of Bif. Chaos 27( 4), 1750063 (2017).

8. Nazaimehr, F., Saaeidi, B., Jafari, S., Sprott, J.C. Are Perpetual Points Sufficient for Locating Hidden Attractors?, Int. J. Bif. Chaos 27(3), 1750037 (2017).

9. Nazaimehr, F., Jafari, S., Golpayegani, S. M. R. H., Sprott, J. C. Categorizing Chaotic Flows from the Viewpoint of Fixed Points and Perpetual Points, Int. J. Bif. Chaos 27(2), 1750023 (2017). 
10. Dudkowski, D., Prasad, A., Kapitaniak, T. Describing chaotic attractors: Regular and perpetual points, Chaos 28(3), 033604 (2018).

11. Kuznetsov, N.V., Leonov, G. A., Mokaev, T.N., Prasad, A., Shrimali, M.D. Finite-time Lyapunov dimension and hidden attractor of the Rabinovich system, Nonlinear Dyn. 92, pp. 267-285 (2018).

12. Chowdhurry, S.N., Ghosh, D. Hidden attractors: A new chaotic system without equilibria, Eur. Phys. J. Special Top. 229, pp. 1299-1308 (2020).

13. Yu, F., Zhang, Z., Liu, L., Shen, H., Huang, Y., Shi, C., Cai, S., Song, Y., Du, S., Xu, Q. Secure Communication Scheme Based on a New 5D Multistable Four-Wing Memristive Hyperchaotic System with Disturbance Inputs, Complexity 2020, 5859273 (2020).

14. Jafari, S., Nazaimehr, F., Sprott, J. C., Golpayegani, S. M. R. H. Limitation of perpetual points for confirming conservation in dynamical systems," Int. J. Bif. Chaos, 25, 1550182 (2015).

15. Cang, S., Wu, A., Wang, Z., Chen, Z. Four-dimensional autonomous dynamical systems with conservative flows: two-case study, Nonlinear Dyn. 89, pp. 2495-2508 (2017).

16. Brzeski, P., Virgin, L. N. System identification of energy dissipation in a mechanical model undergoing high velocities: An indirect use of perpetual points, Mech. Sys. Signal Proces. 108: pp. 115-125 (2018).

17. Wu, A., Cang, S., Zhang, R., Wang, Z., Chen, Z. Hyperchaos in a Conservative System with Nonhyperbolic Fixed Points, Complexity 2018, 9430637 (2018). 
18. Wang, N., Zhang, G., Bao, H. Infinitely many coexisting conservative flows in a 4D conservative system inspired by LC circuit, Nonlinear Dyn. 99, pp. 31973216 (2020).

19. Georgiades, F. Theorem and observation about the nature of perpetual points in conservative mechanical systems, In: Kovacic, I., Lenci, S. (eds) IUTAM Symposium on Exploiting NonLinear Dynamics for Engineering Systems. ENOLIDES 2018. IUTAM Bookseries, vol. 37, pp. 91-104. Springer, Cham (2020).

20. Georgiades, F. Perpetual points in natural dissipative with viscous damping mechanical systems: A theorem and a remark, Proc. of IMechE: Part-C J. of Mech. Eng. Sci (online first 2020). Doi: 10.1177/0954406220934833

21. Georgiades, F. Augmented perpetual manifolds and perpetual mechanical systems-Part I: Definitions, theorem and corollary for triggering perpetual manifolds, application in reduced order modeling and particle-wave motion of flexible mechanical systems, ASME Journal of Computational and Nonlinear Dynamics (accepted for publication).https://doi.org/10.1115/1.4050554

22. Georgiades, F. Augmented perpetual manifolds, a corollary: Dynamics of natural mechanical systems with eliminated internal forces, Proceedings of NODYCON21, Rome, NODYCON, 2021, Springer (accepted for publication, in production).

23. Kusaka, I. Statistical Mechanics for Engineers, Springer International Publishing Switzerland (2015).

24. https://www.nobelprize.org/prizes/chemistry/1977/summary/ 
25. Demtroder, W. Mechanics and Thermodynamics, Springer International Publishing, Switzerland (2017).

26. Wong, K-F V. Thermodynamics for Engineers, CRC Press LLC, Boca Raton, Florida (2000).

27. Meirovitch, L. Fundamental of Vibrations, Long Grove, IL: Waveland Press (2001).

28. Geradin, M, Rixen, D. Mechanical Vibrations, $2^{\text {nd }}$ ed. John Wiley \& Sons, Inc (1997).

29. Meirovitch, L. Methods of Analytical Dynamics, DOVER PUBLICATIONS, INC.: Minessota, New York (1998).

30. Panagiotopoulos, P.D., Glocker, C. Analytical Mechanics. Addendum I: Inequality Constraints with Elastic Impacts. The Convex Case, ZAMM 78(4), pp.219-229 (1998).

31. Leine, R.I., Aeberhard, U., Glocker, C. Hamilton's Principle as Variational Inequality for Mechanical Systems with Impact, J Nonlinear Sci 19, pp. 633664 (2009).

32. Leine, R I, Nijmeier, H. Dynamics and Bifurcations of Non-Smooth Mechanical Systems, Springer-Verlag Berlin Heidelberg (2004). Doi: 10.1007/978-3-54044398-8.

33. Lbach, H, Luth, H. Solid-State Physics An Introduction to Principles of Materials Science, $4^{\text {th }}$ ed. Springer-Verlag, Berlin Heidelberg (2009).

34. Leine, R I., Van de Wouw N. Stability and Convergence of Mechanical Systems with Unilateral Constraints, Springer-Verlag, Berlin, Heidelberg (2008). Doi: 10.1007/978-3-540-76975-0. 
35. Hildebrandt, T H., Introduction to the theory of integration, vol. XIII.

Academic Press Inc. New York (1963).

36. The Scilab Team, version 5.5.2 (2015). http://scilab.org 


\section{APPENDIX-A}

The solutions in exact augmented perpetual manifolds for two types of external forces provided in [21] in the following Table A.1 are presented.

\begin{tabular}{|c|c|c|c|c|}
\hline $\mathrm{r}$ & $\begin{array}{l}\text { External forces } \\
\qquad F_{k}^{(r)}(t)\end{array}$ & Velocity & Displacement & $\begin{array}{l}\text { Wave velocity } \\
\qquad w v_{r}(t)\end{array}$ \\
\hline 1 & $\begin{array}{l}\eta \cdot t+c, \text { with } \\
(\eta, c) \in \mathbb{R}^{2}, \\
\text { (A.1a) }\end{array}$ & $\begin{array}{l}\frac{\eta}{2 \cdot \sum_{j=1}^{N} M_{k, j}} \cdot\left(t^{2}-t_{0}^{2}\right)+\frac{c}{\sum_{j=1}^{N} M_{k, j}} \\
\cdot\left(t-t_{0}\right)+ \\
+\dot{x}_{a, 1}\left(t_{0}\right),(\text { A. } 2 \mathrm{a})\end{array}$ & $\begin{array}{c}\frac{\eta}{6 \cdot \sum_{j=1}^{N} M_{k, j}} \cdot\left(t^{3}-t_{0}^{3}\right)+\frac{c}{2 \cdot \sum_{j=1}^{N} M_{k, j}} \\
-\left(\frac{\eta \cdot t_{0}^{2}}{2 \cdot \sum_{j=1}^{N} M_{k, j}}+\frac{c \cdot t_{0}}{\sum_{j=1}^{N} M_{k, j}}-\dot{x}_{a, 1}^{2}\right)- \\
\left.\cdot\left(t-t_{0}\right)\right) \\
+x_{a, 1}\left(t_{0}\right),(\text { A.3a })\end{array}$ & $\begin{array}{l}\text { Not expected for such } \\
\text { curvilinear form of } \\
\text { displacement }\end{array}$ \\
\hline 2 & $\begin{array}{l}A_{e x} \\
\sin \left(\omega_{e x} \cdot t+\right. \\
\left.\theta_{e x}\right), \text { with } \\
\left(A_{e x}, \theta_{e x}\right) \in \\
\mathbb{R}^{2} \text { and, } \\
\omega_{e x} \in \mathbb{R}_{\geq 0}, \\
\text { (A.1b) }\end{array}$ & $\begin{array}{l}-\frac{A_{e x}}{\sum_{j=1}^{N} M_{k, j} \cdot \omega_{e x}} \cdot \cos \left(\omega_{e x} \cdot t+\theta_{e x}\right)+ \\
+\frac{A_{e x} \cdot \cos \left(\omega_{e x} \cdot t_{0}+\theta_{e x}\right)}{\sum_{j=1}^{N} M_{k, j^{\prime}} \omega_{e x}}+\dot{x}_{a, 2}\left(t_{0}\right),(\text { A. } 2 \mathrm{~b})\end{array}$ & $\begin{array}{l}-\frac{A_{e x}}{\sum_{j=1}^{N} M_{k, j} \cdot \omega_{e x}^{2}} \cdot \sin \left(\omega_{e x} \cdot t+\theta_{e x}\right)+ \\
+\left(\frac{A_{e x} \cdot \cos \left(\omega_{e x} \cdot t_{0}+\theta_{e x}\right)}{\sum_{j=1}^{N} M_{k, j} \cdot \omega_{e x}}+\dot{x}_{a, 2}\left(t_{0}\right)\right) \\
\cdot\left(t-t_{0}\right)+\frac{A_{e x} \cdot \sin \left(\omega_{e x} \cdot t_{0}+\theta_{e x}\right)}{\sum_{j=1}^{N} M_{k, j} \cdot \omega_{e x}^{2}}+x_{a, 2}\left(t_{0}\right), \\
\text { (A.3b) }\end{array}$ & $\begin{array}{l}\frac{A_{e x} \cdot \cos \left(\omega_{e x} \cdot t_{0}+\theta_{e x}\right)}{\sum_{j=1}^{N} M_{k, j^{\prime}} \omega_{e x}}+ \\
\dot{x}_{a, 2}\left(t_{0}\right) \\
\text { (A.4) }\end{array}$ \\
\hline
\end{tabular}

Table A.1. Forms of external forces with explicit equations defining the velocities, the displacements and the wave velocities in the exact augmented perpetual manifolds. 


\section{APPENDIX-B}

In this appendix, the nonsmooth equations of motion of the mechanical system in $\S 3.2$ are written in a certain form for their numerical solution. Since they have differential inclusion, the algorithm for two switch hypersurface boundary functions of [21] based on the switch model developed in [32], is used. Considering the equations of motion (45a-e) with the following change of variables,

$$
s_{1}=r_{1}, s_{2}=r_{2}, s_{3}=\dot{r}_{1}, s_{4}=\dot{r}_{2} .
$$

lead to the following $1^{\text {st }}$-order system of differential equations,

$$
\left\{\dot{s}_{i}\right\}=\left\{\begin{array}{l}
S_{1} \\
S_{2} \\
S_{3} \\
S_{4}
\end{array}\right\}=\left\{\begin{array}{c}
s_{3} \\
s_{4} \\
G_{1}+F_{n s, r_{1}} \\
G_{2}+F_{n s, r_{2}}
\end{array}\right\} \text {, with } i=1,2, \ldots, 4,
$$

with,

$$
\begin{gathered}
G_{1}=\frac{1}{m_{r, 1}} \cdot\left(k_{\mathrm{r}} \cdot\left(s_{2}-s_{1}\right)+k_{n l, r} \cdot\left(s_{2}-s_{1}\right)^{3}+c_{r} \cdot\left(s_{4}-s_{3}\right)+\right. \\
+c_{n l, r} \cdot \tanh \left(b \cdot\left(s_{4}-s_{3}\right)\right)+g_{1} \cdot\left(s_{2}-s_{1}\right)^{2} \cdot\left(s_{4}-s_{3}\right)- \\
\left.-\alpha \cdot s_{3}-\mu \cdot s_{1}-\mu_{n l} \cdot s_{1}^{3}+A_{e x} \cdot \sin \left(\omega_{e x} \cdot t\right)\right), \\
G_{2}=\frac{1}{m_{r, 2}} \cdot\left(k_{\mathrm{r}} \cdot\left(s_{1}-s_{2}\right)+k_{n l, r} \cdot\left(s_{1}-s_{2}\right)^{3}+c_{r} \cdot\left(s_{3}-s_{4}\right)+\right. \\
\quad+c_{n l, r} \cdot \tanh \left(b \cdot\left(s_{3}-s_{4}\right)\right)+g_{1} \cdot\left(s_{2}-s_{1}\right)^{2} \cdot\left(s_{3}-s_{4}\right)- \\
\left.-\frac{m_{r, 2}}{m_{r, 1}} \cdot \alpha \cdot s_{4}-\frac{m_{r, 2}}{m_{r, 1}} \cdot \mu \cdot s_{2}-\frac{m_{r, 2}}{m_{r, 1}} \cdot \mu_{n l} \cdot s_{2}^{3}+\frac{m_{r, 2}}{m_{r, 1}} \cdot A_{e x} \cdot \sin \left(\omega_{e x} \cdot t\right)\right),
\end{gathered}
$$

and,

$$
F_{n s, r_{1}} \in \begin{cases}-\frac{\mu_{1}^{s, r} \cdot F_{1}^{N, r}}{1+\delta \cdot s_{3}}, & s_{3}>0 \\ \mu_{1}^{s, r} \cdot F_{1}^{N, r} \cdot[-1,1], & s_{3}=0 \\ \frac{\mu_{1}^{s, r} \cdot F_{1}^{N, r}}{1-\delta \cdot s_{3}}, & s_{3}<0\end{cases}
$$

and,

$$
F_{n s, r_{2}} \in\left\{\begin{array}{lc}
-\frac{m_{r, 2}}{m_{r, 1}} \cdot \frac{\mu_{1}^{s, r} \cdot F_{1}^{N, r}}{1+\delta \cdot s_{4}}, & s_{4}>0 \\
\frac{m_{r, 2}}{m_{r, 1}} \cdot \mu_{1}^{s, r} \cdot F_{1}^{N, r} \cdot[-1,1], s_{4}=0 & s_{4}<0 \\
\frac{m_{r, 2}}{m_{r, 1}} \cdot \frac{\mu_{1}^{s, r} \cdot F_{1}^{N, r}}{1-\delta \cdot s_{4}}, & .
\end{array}\right.
$$


Considering equations (45d-e) that define the two switch hypersurface boundary functions (HBF), with the variables defined by equation (B.1) the two HBF are given by,

$$
\begin{aligned}
& h_{1}\left(s_{1}, s_{2}, s_{3}, s_{4}\right)=s_{3}, \text { for } \quad \Sigma_{1}, \\
& h_{2}\left(s_{1}, s_{2}, s_{3}, s_{4}\right)=s_{4}, \text { for } \quad \Sigma_{2} .
\end{aligned}
$$

Convexification of the friction force $\left(F_{n s, r_{1}}\right)$ lead to,

$$
F_{n s, r_{1}} \in \begin{cases}-\frac{\mu_{1}^{s, r} \cdot F_{1}^{N, r}}{1+\delta \cdot s_{3}}, & s_{3}>0 \\ \mu_{1}^{s, r} \cdot F_{1}^{N, r} \cdot[-1,1], & s_{3}=0 \\ \frac{\mu_{1}^{s, r} \cdot F_{1}^{N, r}}{1-\delta \cdot s_{3}}, & s_{3}<0\end{cases}
$$

therefore,

$$
S_{3,+}=G_{1}-\frac{\mu_{1}^{s, r} \cdot F_{1}^{N, r}}{m_{r, 1} \cdot\left(1+\delta \cdot s_{3}\right)}
$$

and,

$$
S_{3,-}=G_{1}+\frac{\mu_{1}^{s, r} \cdot F_{1}^{N, r}}{m_{r, 1} \cdot\left(1-\delta \cdot S_{3}\right)} .
$$

The convexification of the other friction force leads to,

$$
F_{n s, r_{2}} \in\left\{\begin{array}{lc}
-\frac{m_{r, 2}}{m_{r, 1}} \cdot \frac{\mu_{1}^{s, r} \cdot F_{1}^{N, r}}{1+\delta \cdot s_{4}}, & s_{4}>0 \\
\frac{m_{r, 2}}{m_{r, 1}} \cdot \mu_{1}^{s, r} \cdot F_{1}^{N, r} \cdot[-1,1], s_{4}=0, & s_{4}<0 \\
\frac{m_{r, 2}}{m_{r, 1}} \cdot \frac{\mu_{1}^{s, r} \cdot F_{1}^{N, r}}{1-\delta \cdot s_{4}}, & s^{N},
\end{array}\right.
$$

therefore,

$$
\begin{aligned}
& S_{4,+}=G_{2}-\frac{\mu_{1}^{s, r} \cdot F_{1}^{N, r}}{m_{r, 1} \cdot\left(1+\delta \cdot s_{4}\right)}, \\
& S_{4,-}=G_{2}+\frac{\mu_{1}^{s, r} \cdot F_{1}^{N, r}}{m_{r, 1} \cdot\left(1-\delta \cdot s_{4}\right)} .
\end{aligned}
$$

Finally the two normal vectors to each one of the switch hypersurfaces are given by,

$$
\begin{aligned}
& n_{1}^{T}=\left[\begin{array}{llll}
\frac{\partial h_{1}}{\partial s_{1}} & \frac{\partial h_{1}}{\partial s_{2}} & \frac{\partial h_{1}}{\partial s_{3}} & \frac{\partial h_{1}}{\partial s_{4}}
\end{array}\right]^{T}=\left[\begin{array}{lllll}
0 & 0 & 1 & 0
\end{array}\right]^{T}, \text { for } \quad \Sigma_{1} \\
& n_{2}^{T}=\left[\begin{array}{llllll}
\frac{\partial h_{2}}{\partial s_{1}} & \frac{\partial h_{2}}{\partial s_{2}} & \frac{\partial h_{2}}{\partial s_{3}} & \frac{\partial h_{2}}{\partial s_{4}}
\end{array}\right]^{T}=\left[\begin{array}{lllll}
0 & 0 & 0 & 1
\end{array}\right]^{T}, \text { for } \quad \Sigma_{2} .
\end{aligned}
$$

In switch model the identification of regions that the system belongs is found with the multiplication of the normal vectors with the vector fields. Simplifying things the explicit form is, 


$$
n_{1}^{T} \times\left\{\begin{array}{l}
S_{1} \\
S_{2} \\
S_{3} \\
S_{4}
\end{array}\right\}=S_{3} .
$$

Therefore, convexification of the vector field using the switch model near the surface $\Sigma_{1}$ should be done only in the $S_{3}$ term of the vector field using the parameter,

$$
a_{1}=\frac{S_{3,-}+\tau^{-1} \cdot h_{1}}{S_{3,-}-S_{3,+}}
$$

Also, for the other hypersurface boundary,

$$
n_{2}^{T} \times\left\{\begin{array}{l}
S_{1} \\
S_{2} \\
S_{3} \\
S_{4}
\end{array}\right\}=S_{4} .
$$

Therefore, the convexification of the vector field using the switch model near the surface $\Sigma_{2}$ must be done only in $S_{4}$ term of the vector field using the parameter,

$$
a_{2}=\frac{S_{4,-}+\tau^{-1} \cdot h_{2}}{S_{4,-}-S_{4,+}}
$$

The aforementioned definitions are sufficient for the numerical solution of the 2dof nonsmooth system with the application of the double switch algorithm given in Figure A.1 of Appendix A in [21]. 